\title{
SOLUTIONS TO A SIMPLIFIED INITIAL BOUNDARY VALUE PROBLEM FOR 1D HYPERBOLIC EQUATION WITH INTERIOR DEGENERACY
}

\author{
Vladimir L. Borsch, Peter I. Kogut ${ }^{\dagger}$ \\ Communicated by Prof. G. Leugering
}

\begin{abstract}
A 1-parameter initial boundary value problem (IBVP) for a linear homogeneous degenerate wave equation (JODEA, 28(1), 1 в ““ 42) in a space-time rectangle is considered. The origin of degeneracy is the power law coefficient function with respect to the spatial distance to the symmetry line of the rectangle, the exponent being the only parameter of the problem, ranging in $(0,1)$ and $(1,2)$ and producing the weak and strong degeneracy respectively. In the case of weak degeneracy separation of variables is used in the rectangle to obtain the unique bounded continuous solution to the IBVP, having the continuous flux. In the case of strong degeneracy the IBVP splits into the two derived IBVPs posed respectively in left and right half-rectangles and solved separately using separation of variables. Continuous matching of the obtained left and right families of bounded solutions to the IBVPs results in a linear integro-differential equation of convolution type. The Laplace transformation is used to solve the equation and obtain a family of bounded solutions to the IBVP, having the continuous flux and depending on one undetermined function..
\end{abstract}

Key words: degenerate wave equation, separation of variables, linear integro-differential equation of convolution type, Laplace transformation.

2010 Mathematics Subject Classification: 35L05, 35L35, 35L80.

\section{Introduction and Setting of the Problem}

The current study is a sequel to [2] and deals with the following 1-parameter simplified initial boundary value problem (IBVP) for the degenerate wave equation in the space-time rectangle $[0, T] \times[-1,+1]$

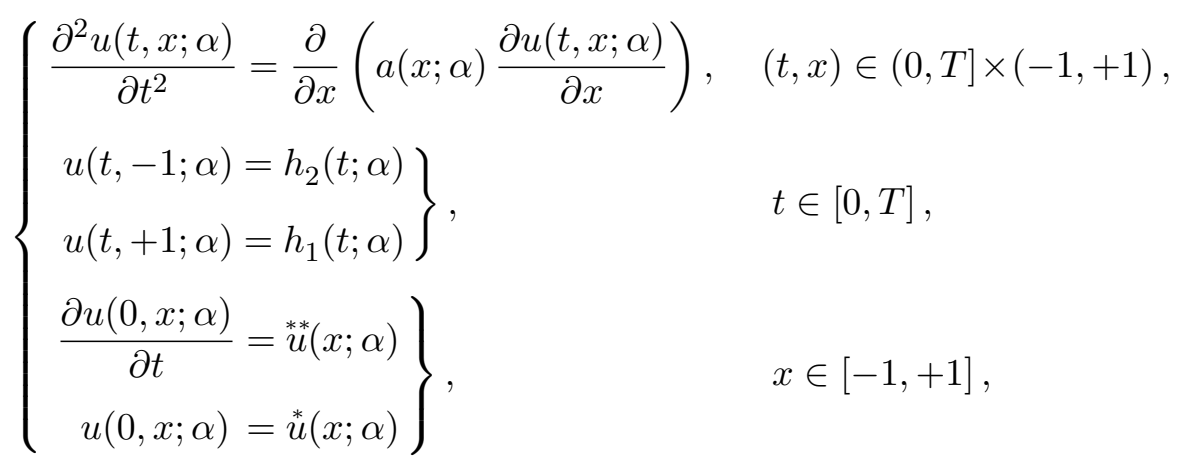

*Dept. of Differential Equations, Faculty of Mech \& Math, Oles Honchar Dnipro National University, 72, Gagarin av., Dnipro, 49010, Ukraine, bvl@dsu.dp.ua

${ }^{\dagger}$ Dept. of Differential Equations, Faculty of Mech \& Math, Oles Honchar Dnipro National University, 72, Gagarin av., Dnipro, 49010, Ukraine, p.kogut@i.ua

(C) V.L. Borsch, P. I. Kogut, 2021. 
where known control functions $h_{1}(t ; \alpha), h_{2}(t ; \alpha) \in \mathscr{C}^{1}[0, T] \cap \mathscr{C}^{2}(0, T]$ obey the compatibility conditions: $h_{1}(0 ; \alpha)=\stackrel{*}{u}(+1 ; \alpha), h_{1}^{\prime}(0 ; \alpha)=* *(+1 ; \alpha), h_{2}(0 ; \alpha)=\stackrel{*}{u}(-1 ; \alpha)$, and $h_{2}^{\prime}(0 ; \alpha)=\stackrel{* *}{u}(-1 ; \alpha)$, and the 1-parameter family of coefficient functions is defined as follows

$$
a(x ; \alpha)=|x|^{\alpha}, \quad x \in[-1,+1],
$$

the parameter $\alpha \in(0,2)$, and all the dependent and independent variables are nondimensional. Simplification of the IBVP (1.1) compared to that of [2] is in extending the power law for the coefficient function to the segment $[-1,+1]$. One should refer to [2] to find out more details on the problem formulation.

The degenerate wave equation of the IBVP (1.1) has non-unique one-sided solutions, obtained in [2] as the following power series

$$
\left\{\begin{array}{l}
u_{1, j}(t, x ; \alpha)=\sum_{\gamma=0}^{\infty} U_{1, j, \gamma}(t ; \alpha)|x|^{\gamma \theta} \\
u_{3, j}(t, x ; \alpha)=U_{3, j}(t ; \alpha)+|x|^{\nu} U_{3, j, 0}(t ; \alpha), \\
u_{5, j}(t, x ; \alpha)=U_{5, j}(t ; \alpha)+|x|^{\nu} \sum_{\gamma=0}^{\infty} U_{5, j, \gamma}(t ; \alpha)|x|^{\gamma \theta}
\end{array}\right.
$$

where $\nu=1-\alpha, \theta=2-\alpha$, the values $\{1,3,5\}$ of the first subscript $k$ refer to the kind of the above solutions, and the values $\{1,2\}$ of the second subscript $j$ refer to the values $x>0$ and $x<0$ respectively. The coefficient functions of the solutions $u_{1, j}(t, x ; \alpha)$ and $u_{5, j}(t, x ; \alpha)$ obey the following recurrence relations

$$
\left\{\begin{array}{l}
U_{1, j, \gamma-1}^{\prime \prime}(t ; \alpha)=\gamma \theta[\gamma \theta-\nu] U_{1, j, \gamma}(t ; \alpha), \\
U_{5, j, \gamma-1}^{\prime \prime}(t ; \alpha)=\gamma \theta[\gamma \theta+\nu] U_{5, j, \gamma}(t ; \alpha),
\end{array} \quad \gamma \in \mathbb{N} .\right.
$$

and the function $U_{5, j}(t ; \alpha)$ is linear: $U_{5, j}^{\prime \prime}(t ; \alpha)=0$, whereas both coefficient functions $U_{3, j}(t ; \alpha), U_{3, j, 0}(t ; \alpha)$ are linear: $U_{3, j}^{\prime \prime}(t ; \alpha)=0, U_{3, j, 0}^{\prime \prime}(t)=0$. Note, that the solution of the third kind is derived from the fifth one when $U_{5, j, 0}^{\prime \prime}(t ; \alpha)=0$.

The solution of the first kind is bounded for $\alpha \in(0,2)$, whereas the solutions of the third and fifth kind are bounded for $\alpha \in(0,1]$ and unbounded for $\alpha \in(1,2)$. An other representation of the solutions of the first and fifth kinds, showing their relation to the Bessel functions of the first kind and orders $\mp \varrho$, reads as follows

$$
\left\{\begin{array}{l}
u_{1, j}(t, x ; \alpha)=|x|^{\frac{\nu}{2}}\left(s^{-\varrho} \sum_{\gamma=0}^{\infty} U_{1, j, \gamma}(t ; \alpha) s^{2 \gamma}\right), \\
u_{5, j}(t, x ; \alpha)=U_{5, j}(t ; \alpha)+|x|^{\frac{\nu}{2}}\left(s^{+\varrho} \sum_{\gamma=0}^{\infty} U_{5, j, \gamma}(t ; \alpha) s^{2 \gamma}\right),
\end{array}\right.
$$

where $\varrho \theta=\nu$ and the auxiliary variable $s=|x|^{\frac{\theta}{2}}$ is used. 
The spatial derivatives of the solutions (1.3)

$$
q_{k, j}(t, x ; \alpha)=\frac{\partial u_{k, j}}{\partial x}=\operatorname{sign}(x)\left\{\begin{array}{l}
|x|^{\nu} \theta \sum_{\gamma=1}^{\infty} \gamma U_{1, j, \gamma}(t ; \alpha)|x|^{(\gamma-1) \theta}, \\
|x|^{-\alpha} \nu U_{3, j, 0}(t ; \alpha), \\
|x|^{-\alpha} \sum_{\gamma=0}^{\infty}[\nu+\gamma \theta] U_{5, j, \gamma}(t ; \alpha)|x|^{\gamma \theta}
\end{array}\right.
$$

are bounded in the case of weak degeneracy $(\alpha \in(0,1))$ and unbounded in the case of strong degeneracy $(\alpha \in(1,2))$ for the first kind, and always unbounded for the third and fifth kinds. The fluxes of the solutions (1.3)

$$
-f_{k, j}(t, x ; \alpha)=a q_{k, j}=\left\{\begin{array}{c}
x \theta \sum_{\gamma=1}^{\infty} \gamma U_{1, j, \gamma}(t ; \alpha)|x|^{(\gamma-1) \theta} \\
\operatorname{sign}(x) \nu U_{3, j, 0}(t ; \alpha), \\
\operatorname{sign}(x) \sum_{\gamma=0}^{\infty}[\nu+\gamma \theta] U_{5, j, \gamma}(t ; \alpha)|x|^{\gamma \theta}
\end{array}\right.
$$

are bounded, but have quite different nature at the interior degeneracy: the flux of the first kind is vanishing at the degeneracy and, therefore, continuous; whereas the two others have generally non-vanishing values of opposite signs. The oddbehavior of the one-sided fluxes of the fifth kind prompts us the way of their continuous matching.

In the current study we shall try to continuously match the one-sided solutions (1.3) of the first and fifth kinds (therefore, the subscript $k$ takes values $\{1,5\}$ ) to find bounded solutions to the IBVP (1.1) using the method of separation of variables (SV) and implying an analogy of the required solutions with a continuous imaginary 'string'. The current study is arranged as follows.

In Section 2 we: 1) give some preliminaries on SV in relation to the original IBVP in the case of weak degeneracy and based on the one-sided solutions of kinds 1,5, both continuous and improving to have the continuous fluxes; 2) split the original IBVP posed in the space-time rectangle $[0, T] \times[-1,+1]$ and describing the behavior of the continuous 'string', into the derived $\mathrm{IBVP}_{2}$ posed in the left space-time rectangle $[0, T] \times[-1,0]$ ) and the IBVP $_{1}$ posed in the right space-time rectangle $[0, T] \times[0,+1])$, describing respectively the behaviour of the left and the right parts of the 'string' separately in the case of strong degeneracy; 3 ) formulate the conditions for continuous matching the bounded solutions $u_{1, j}(t, x ; \alpha)$ to the $\operatorname{IBVP}_{j}$ and expressing the integrity of the 'string' and continuity of the flux; 4) apply the method of SV to find the unique bounded solutions $u(t, x ; \alpha)$ to the IBVP in the case of weak degeneracy, continuous and having 
the continuous flux; 5) apply the method of SV to find families of bounded solution $u_{1, j}(t, x ; \alpha)$ to the $\mathrm{IBVP}_{j}$ in the case of strong degeneracy, having the continuous flux and depending on undetermined functions $h_{j+2}(t ; \alpha) \in \mathscr{C}^{1}[0, T] \bigcap \mathscr{C}^{2}(0, T]$; 6) apply the continuity condition to the solutions $u_{1, j}(t, x ; \alpha)$ to derive a linear integro-differential equation of convolution type with respect to the required functions $h_{j+2}(t ; \alpha)$.

In Section 3 we solve the above integro-differential equation with respect to the difference $h_{3}(t ; \alpha)-h_{4}(t ; \alpha)$ and show that one of the two functions can be chosen quite freely, that is, the bounded solutions to the IBVP of the resulting family are continuous and have the continuous fluxes.

In Section 4 we summarize the results obtained and some observations on the procedures applied.

In Section 2 we place some useful rules to calculate the coefficients of expansions in the series of the eigenfunctions used in Section 2.

\section{Method of Separation of Variables}

\subsection{Preliminaries to SV}

Implementing SV to the IBVP (1.1) is essentially based on the following two assertions.

Proposition 2.1. Let the following incomplete 1-parameter boundary value problems be given

$$
\left\{\begin{array}{l}
{\left[a(x ; \alpha) Z_{j}^{\prime}(x ; \alpha)\right]^{\prime}+\lambda_{j}(\alpha) Z_{j}(x ; \alpha)=0, \quad 0<|x|<1,} \\
Z_{j}(\mp 1 ; \alpha)=0
\end{array}\right.
$$

then: 1) the eigenvalues and the eigenfunctions of the problems of the two kinds $\left(\lambda_{k, j, \mu}(\alpha), Z_{k, j, \mu}(x ; \alpha)\right) \equiv\left(\lambda_{k, \mu}(\alpha), Z_{k, \mu}(x ; \alpha)\right)$ (marked with the first subscript $k \in\{1,5\})$ are defined as follows

$$
\begin{cases}\lambda_{1, \mu}(\alpha)=\left(\frac{\theta}{2} s_{1, \mu}\right)^{2} \equiv \sigma_{1, \mu}^{2}, & Z_{1, \mu}(x ; \alpha)=|x|^{\frac{\nu}{2}} \mathrm{~J}_{-\varrho}\left(s_{1, \mu}|x|^{\frac{\theta}{2}}\right), \\ \lambda_{5, \mu}(\alpha)=\left(\frac{\theta}{2} s_{5, \mu}\right)^{2} \equiv \sigma_{5, \mu}^{2}, & Z_{5, \mu}(x ; \alpha)=|x|^{\frac{\nu}{2}} \mathrm{~J}_{+\varrho}\left(s_{5, \mu}|x|^{\frac{\theta}{2}}\right)\end{cases}
$$

where $\nu, \theta, \varrho$ are the $\alpha$-dependent quantities

$$
\nu(\alpha)=1-\alpha, \quad \theta(\alpha)=2-\alpha, \quad \varrho(\alpha)=\frac{\nu}{\theta}=\frac{1-\alpha}{2-\alpha}
$$

$\mathrm{J}_{\mp \varrho}(s)$ are the Bessel functions of the first kind and orders $\mp \varrho[7] ;\left\{s_{k, \mu}\right\}_{\mu=1}^{\infty}$ are the unbounded monotonically increasing sequences of the zeros of functions 
$\left.\mathrm{J}_{\mp \varrho}(s) ; 2\right)$ the eigenfunctions $(2.2)$ of each kind are orthogonal in $\mathscr{L}_{2}(-1,0)$ and $\mathscr{L}_{2}(0,+1)$ respectively, that is

$$
\mp \int_{0}^{\mp 1} Z_{k, \mu}(x ; \alpha) Z_{k, \gamma}(x ; \alpha) \mathrm{d} x=\frac{1}{\theta} \mathrm{J}_{\mp \varrho+1}^{2}\left(s_{k, \mu}\right) \delta_{\mu, \gamma} \equiv\left\|Z_{k, \mu}\right\|^{2} \delta_{\mu, \gamma},
$$

where $\mu, \gamma \in \mathbb{N}$, and $\delta_{\mu, \gamma}$ is the Kronecker delta.

Proof. We start proving the first part of the proposition from representing the eigenfunctions (2.2) in such a generic formulation

$$
Z_{k, \mu}(x ; \alpha)=|x|^{\frac{\nu}{2}} \mathrm{Z}_{\varrho}(s), \quad s=s_{k, \mu}|x|^{\frac{\theta}{2}},
$$

where the generic Bessel function $\mathrm{Z}_{\varrho}(s)$ stands for the Bessel functions $\mathrm{J}_{\mp \varrho}(s)$ of the first kind and satisfies the ordinary differential equation

$$
s^{2} \mathrm{Z}_{\varrho}^{\prime \prime}(s)+s \mathrm{Z}_{\varrho}^{\prime}(s)+\left(s^{2}-\varrho^{2}\right) \mathrm{Z}_{\varrho}(s)=0 .
$$

Differentiating the generic eigenfunction (2.5) with respect to $x$ yields to

$$
\begin{aligned}
Z_{k, \mu}^{\prime}(x ; \alpha) & =\operatorname{sign}(x)\left[\frac{\nu}{2}|x|^{\frac{\nu}{2}-1} \mathrm{Z}_{\varrho}(s)+\frac{\theta}{2} s_{k, \mu}|x|^{\frac{\nu}{2}+\frac{\theta}{2}-1} \mathrm{Z}_{\varrho}^{\prime}(s)\right], \\
a(x ; \alpha) Z_{k, \mu}^{\prime}(x ; \alpha) & =\operatorname{sign}(x)\left[\frac{\nu}{2}|x|^{-\frac{\nu}{2}} \mathrm{Z}_{\varrho}(s)+\frac{\theta}{2} s_{k, \mu}|x|^{-\frac{\nu}{2}+\frac{\theta}{2}} \mathrm{Z}_{\varrho}^{\prime}(s)\right], \\
{\left[a(x ; \alpha) Z_{k, \mu}^{\prime}(x ; \alpha)\right]^{\prime} } & =|x|^{-\frac{\nu}{2}-1}\left[-\left(\frac{\nu}{2}\right)^{2} \mathrm{Z}_{\varrho}(s)+\left(\frac{\theta}{2}\right)^{2}\left(s^{2} \mathrm{Z}_{\varrho}^{\prime \prime}(s)+s \mathrm{Z}_{\varrho}^{\prime}(s)\right)\right] \\
& =-\left(\frac{\theta}{2} s_{k, \mu}\right)^{2}|x|^{\frac{\nu}{2}} \mathrm{Z}_{\varrho}(s)=-\sigma_{k, \mu}^{2} Z_{k, \mu}(x),
\end{aligned}
$$

wherefrom we conclude, that the functions (2.2) indeed satisfy the differential equation of the problems (2.1). This completes the proof of the first part of the proposition.

To prove the second part of the proposition, we use:

1) the variable transformation $s=x^{\frac{\theta}{2}}$ when calculating the integral

$$
\int_{0}^{1} Z_{k, \mu}(x ; \alpha) Z_{k, \gamma}(x ; \alpha) \mathrm{d} x=\frac{2}{\theta} \int_{0}^{1} s \mathrm{Z}_{\varrho}\left(s_{k, \mu} s\right) \mathrm{Z}_{\varrho}\left(s_{k, \gamma} s\right) \mathrm{d} s ;
$$

2) the known value of the last integral [7]

$$
\int_{0}^{1} s \mathrm{Z}_{\varrho}\left(s_{k, \mu} s\right) \mathrm{Z}_{\varrho}\left(s_{k, \gamma} s\right) \mathrm{d} s=\frac{1}{2} \mathrm{Z}_{\varrho+1}^{2}\left(s_{k, \mu}\right) \delta_{\mu, \gamma} .
$$

This completes the proof of the second part of the proposition. 
Proposition 2.2. Let the following composite 1-parameter boundary value problem be given

$$
\left\{\begin{array}{l}
{\left[a(x ; \alpha) X^{\prime}(x ; \alpha)\right]^{\prime}+\lambda(\alpha) X(x ; \alpha)=0, \quad 0<|x|<1,} \\
X(\mp 1 ; \alpha)=0,\left.\quad[X(x ; \alpha)]\right|_{x=0-0}=\left.[X(x ; \alpha)]\right|_{x=0+0}, \\
{\left.\left[a(x ; \alpha) X^{\prime}(x ; \alpha)\right]\right|_{x=0-0}=\left.\left[a(x ; \alpha) X^{\prime}(x ; \alpha)\right]\right|_{x=0+0},}
\end{array}\right.
$$

then: 1) in the case of weak degeneracy, the eigenvalues and the eigenfunctions of the problem of the two kinds (marked with the first subscript $k \in\{1,5\}$ ) are defined as follows

$$
\begin{cases}\lambda_{1, \mu}(\alpha)=\sigma_{1, \mu}^{2}, & X_{1, \mu}(x ; \alpha)= \\ \lambda_{5, \mu}(\alpha)=\sigma_{5, \mu}^{2}, & X_{5, \mu}(x ; \alpha)=\operatorname{sign}(x) Z_{5, \mu}(x ; \alpha),\end{cases}
$$

where $\sigma_{k, \mu}^{2}$ and $Z_{k, \mu}(x ; \alpha)$ are given in $(2.2)$ of Proposition $\left.2.1 ; 2\right)$ the eigenfunctions $(2.7)$ of both kinds are orthogonal in $\mathscr{L}_{2}(-1,+1)$, that is

$$
\left\{\begin{array}{l}
\int_{-1}^{+1} X_{k, \mu}(x ; \alpha) X_{k, \gamma}(x ; \alpha) d x=2\left\|Z_{k, \mu}\right\|^{2} \delta_{\mu, \gamma} \equiv\left\|X_{k, \mu}\right\|^{2} \delta_{\mu, \gamma}, \\
\int_{-1}^{+1} X_{1, \mu}(x ; \alpha) X_{5, \gamma}(x ; \alpha) d x=0 .
\end{array}\right.
$$

Proof. From Proposition 2.1 it follows that the functions $X_{k, \mu}(x ; \alpha)(2.7)$ satisfy the differential equation of the boundary value problem, hence, we concentrate our efforts on calculating the one-sided values of $X_{k, \mu}(x ; \alpha)$ and $a(x ; \alpha) X_{k, \mu}^{\prime}(x ; \alpha)$ at the interior degeneracy location. Substituting the known power series [7]

$$
\mathrm{Z}_{\varrho}(s)=\mathrm{J}_{\mp \varrho}(s)=\left(\frac{s}{2}\right)^{\mp \varrho} \sum_{\gamma=0}^{\infty} \frac{(-1)^{\gamma}}{\gamma ! \Gamma(1+\gamma \mp \varrho)}\left(\frac{s}{2}\right)^{2 \gamma}
$$

into (2.5) obtains the series representations for the quantities of interest

$$
\begin{aligned}
& \left\{\begin{array}{l}
X_{1, \mu}(x ; \alpha)=\left(\frac{s_{1, \mu}}{2}\right)^{-\varrho} \sum_{\gamma=0}^{\infty} \frac{(-1)^{\gamma}|x|^{\gamma \theta}}{\gamma ! \Gamma(1-\varrho+\gamma)}\left(\frac{s_{1, \mu}}{2}\right)^{2 \gamma} \\
X_{5, \mu}(x ; \alpha)=\operatorname{sign}(x)|x|^{\nu}\left(\frac{s_{5, \mu}}{2}\right)^{+\varrho} \sum_{\gamma=0}^{\infty} \frac{(-1)^{\gamma}|x|^{\gamma \theta}}{\gamma ! \Gamma(1+\varrho+\gamma)}\left(\frac{s_{5, \mu}}{2}\right)^{2 \gamma}
\end{array}\right. \\
& \left\{\begin{array}{l}
a(x ; \alpha) X_{1, \mu}^{\prime}(x ; \alpha)=\left(\frac{s_{1, \mu}}{2}\right)^{-\varrho} \theta x \sum_{\gamma=1}^{\infty} \frac{(-1)^{\gamma} \gamma|x|^{(\gamma-1) \theta}}{\gamma ! \Gamma(1-\varrho+\gamma)}\left(\frac{s_{1, \mu}}{2}\right)^{2 \gamma} \\
a(x ; \alpha) X_{5, \mu}^{\prime}(x ; \alpha)=\left(\frac{s_{5, \mu}}{2}\right)^{+\varrho} \sum_{\gamma=0}^{\infty} \frac{(-1)^{\gamma}[\nu+\gamma \theta]|x|^{\gamma \theta}}{\gamma ! \Gamma(1+\varrho+\gamma)}\left(\frac{s_{5, \mu}}{2}\right)^{2 \gamma} .
\end{array}\right.
\end{aligned}
$$


The resulting series $(2.10),(2.11)$ yield to the required values

$$
\begin{gathered}
\left\{\begin{array}{cc}
X_{1, \mu}(0 ; \alpha)=\left(\frac{s_{1, \mu}}{2}\right)^{-\varrho} \frac{1}{\Gamma(1-\varrho)}, & \alpha \in(0,2), \\
X_{5, \mu}(0 ; \alpha)=0, & \alpha \in(0,1), \\
\operatorname{sign}(x) \lim _{x \rightarrow 0}|x|^{-\nu} X_{5, \mu}(x ; \alpha)=\left(\frac{s_{5, \mu}}{2}\right)^{+\varrho} \frac{1}{\Gamma(1+\varrho)}, & \alpha \in[1,2),
\end{array}\right. \\
\begin{cases}{\left.\left[a(x ; \alpha) X_{1, \mu}^{\prime}(x ; \alpha)\right]\right|_{x=0}=0,} & \alpha \in(0,2), \\
{\left.\left[a(x ; \alpha) X_{5, \mu}^{\prime}(x ; \alpha)\right]\right|_{x=0}=\left(\frac{s_{5, \mu}}{2}\right)^{\varrho} \frac{\nu}{\Gamma(1+\varrho)} \neq 0,} & \end{cases}
\end{gathered}
$$

and this completes the proof of the first part of the proposition.

Orthogonality of the eigenfunctions of each kind directly follows from Proposition 2.1, therefore, our concern is orthogonality of the eigenfunctions of the different kinds, that can be quite easily proved, indeed,

$$
\begin{aligned}
& \int_{-1}^{+1} X_{1, \mu}(x ; \alpha) X_{5, \gamma}(x ; \alpha) d x \\
= & \int_{-1}^{0} X_{1, \mu}(x ; \alpha) X_{5, \gamma}(x ; \alpha) d x+\int_{0}^{+1} X_{1, \mu}(x ; \alpha) X_{5, \gamma}(x ; \alpha) d x \\
= & -\int_{-1}^{0} Z_{1, \mu}(x ; \alpha) Z_{5, \gamma}(x ; \alpha) d x+\int_{0}^{+1} Z_{1, \mu}(x ; \alpha) Z_{5, \gamma}(x ; \alpha) d x \\
= & -\int_{0}^{+1} Z_{1, \mu}(x ; \alpha) Z_{5, \gamma}(x ; \alpha) d x+\int_{0}^{+1} Z_{1, \mu}(x ; \alpha) Z_{5, \gamma}(x ; \alpha) d x=0 .
\end{aligned}
$$

This completes the proof of the second part of the proposition.

Before implementing the method of SV, we make some notes.

First, to build the eigenfunctions $Z_{5, \mu}(x ; \alpha)$, we use the Bessel functions of the first kind and order $+\varrho$, rather than the proper Neumann functions [7], to simplify our analysis of the IBVP. It means that the integer values of order $-\varrho$

$$
-\varrho=-\frac{1-\alpha}{2-\alpha}=m \in \mathbb{Z} \quad \Leftrightarrow \quad \alpha=\frac{2 m+1}{m+1}
$$

can not be considered, i. e., the values of $\alpha=1, \frac{3}{2}, \frac{5}{3}, \frac{7}{4}$, etc., produced by the values of $m=0,1,2,3$, etc.

Second, to guarantee uniform convergency of the expansions in series of the eigen-functions $Z_{1, \mu}(x ; \alpha), X_{k, \mu}(x ; \alpha)$, based on the Bessel functions $\mathrm{J}_{\mp \varrho}(s)$, we have to impose the following restriction $[6,7]$ on the values of $\varrho$

$$
-\frac{1}{2} \leqslant \varrho=\frac{1-\alpha}{2-\alpha} \leqslant+\frac{1}{2} .
$$


Third, to solve the IBVP (1.1) in the case of weak degeneracy, we apply the bounded eigenfunctions of Prop. 2.2.

Fourth, in the case of weak degeneracy we reduce solving the IBVP (1.1) to the following two-step procedure: 1) solving the derived initial boundary value problems

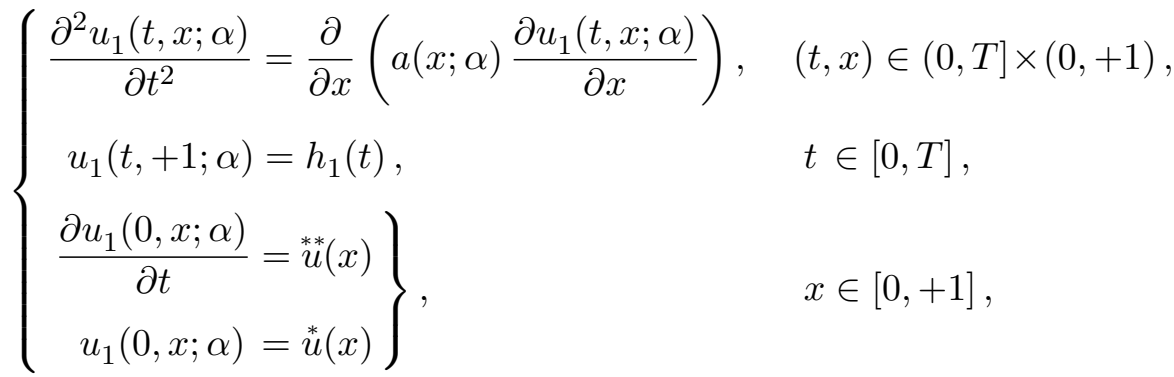

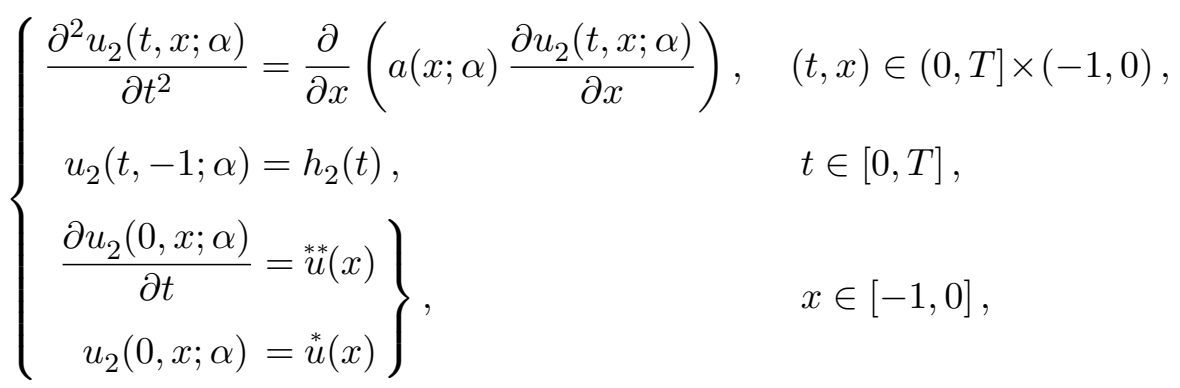

posed in the 'right' $[0, T] \times[0,+1]$ and the 'left' $[0, T] \times[-1,0]$ space-time rectangles and referred to as the $\mathrm{IBVP}_{1}$ and the $\mathrm{IBVP}_{2}$ respectively; 2) matching the solutions $u_{1}(t, x ; \alpha)$ and $u_{2}(t, x ; \alpha)$ to the above initial boundary value problems

$$
u(t, x ; \alpha)= \begin{cases}u_{2}(t, x ; \alpha), & (t, x) \in[0, T] \times[-1,0], \\ u_{1}(t, x ; \alpha), & (t, x) \in[0, T] \times[0,+1],\end{cases}
$$

by imposing the condition of continuity at the degeneracy segment $[0, T] \times\{0\}$

$$
u_{2}(t, 0 ; \alpha)=u_{1}(t, 0 ; \alpha), \quad t \in[0, T] .
$$

When applying the above procedure, we drop the subscript $k$, indicating the first kind of the solutions (1.3), the only one bounded in the case of strong degeneracy, therefore, the only remaining subscript is $j$.

\subsection{Implementing $\mathrm{SV}$ to the IBVP}

In the current section our concern is the bounded solution to the IBVP in the case of weak degeneracy. The required solution is assumed to have the following representation

$$
u(t, x ; \alpha)=v(t, x ; \alpha)+w(t, x ; \alpha),
$$


where: $a)$ the function $v(t, x ; \alpha)$ is also required; $b)$ the function $w(t, x ; \alpha)$ is given as follows

$$
w(t, x ; \alpha)=\phi_{j=2}(x ; \alpha) h_{j=2}(t ; \alpha)+\phi_{j=1}(x ; \alpha) h_{j=1}(t ; \alpha),
$$

c) the smooth blending functions $\phi_{1}(x ; \alpha), \phi_{2}(x ; \alpha)$ satisfy the following boundary and regularity conditions, respectively

$$
\begin{gathered}
\begin{cases}\phi_{1}(+1 ; \alpha)=1, & \phi_{1}(-1 ; \alpha)=0, \\
\phi_{2}(+1 ; \alpha)=0, & \phi_{2}(-1 ; \alpha)=1 ;\end{cases} \\
\left\{\begin{array}{l}
\psi_{1}(x ; \alpha) \equiv \varphi_{1}^{\prime}(x ; \alpha)=\left[a(x ; \alpha) \phi_{1}^{\prime}(x ; \alpha)\right]^{\prime} \in \mathscr{C}[-1,+1], \\
\psi_{2}(x ; \alpha) \equiv \varphi_{2}^{\prime}(x ; \alpha)=\left[a(x ; \alpha) \phi_{2}^{\prime}(x ; \alpha)\right]^{\prime} \in \mathscr{C}[-1,+1] .
\end{array}\right.
\end{gathered}
$$

Combining (2.18) $-(2.20)$ yields to: $a)$ the initial conditions for the required function $v(t, x ; \alpha)$

$$
\left\{\begin{aligned}
v(0, x ; \alpha) & =u(0, x ; \alpha)-w(0, x ; \alpha) \equiv \stackrel{*}{v}(x ; \alpha), \\
\frac{\partial v(0, x ; \alpha)}{\partial t} & =\frac{\partial u(0, x ; \alpha)}{\partial t}-\frac{\partial w(0, x ; \alpha)}{\partial t} \equiv \stackrel{* *}{v}(x ; \alpha),
\end{aligned}\right.
$$

and $b$ ) reformulation of the IBVP into the following one with respect to $v(t, x ; \alpha)$

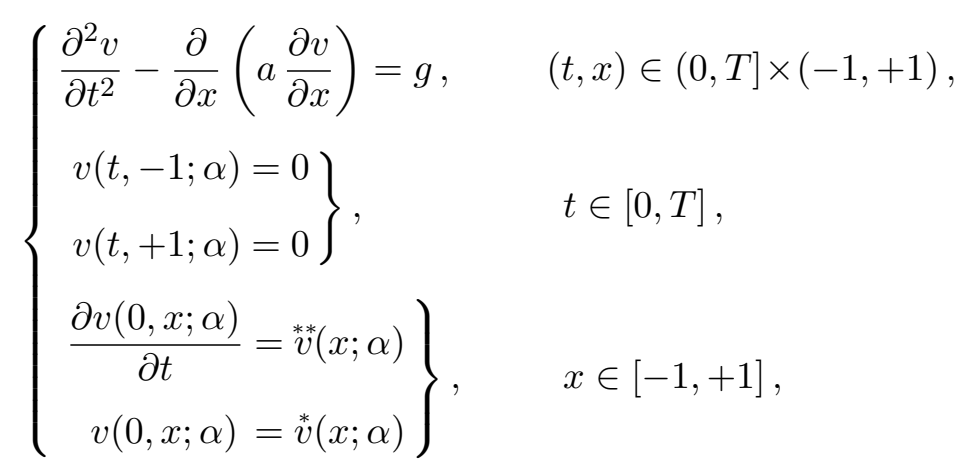

where the right-hand side of the above degenerate wave equation reads

$$
g(t, x ; \alpha)=-\frac{\partial^{2} w(t, x ; \alpha)}{\partial t^{2}}+\frac{\partial}{\partial x}\left(a(x ; \alpha) \frac{\partial w(t, x ; \alpha)}{\partial x}\right) .
$$

Then the initial functions (2.22) and the right-hand side (2.24) are expanded into the series

$$
\left\{\begin{array}{l}
\stackrel{*}{v}(x ; \alpha)=\sum_{\mu=1}^{\infty} v_{1, \mu}^{*}(\alpha) X_{1, \mu}(x ; \alpha)+\sum_{\mu=1}^{\infty} v_{5, \mu}^{*}(\alpha) X_{5, \mu}(x ; \alpha), \\
\stackrel{*}{v}(x ; \alpha)=\sum_{\mu=1}^{\infty} \stackrel{* *}{v}_{1, \mu}(\alpha) X_{1, \mu}(x ; \alpha)+\sum_{\mu=1}^{\infty} \stackrel{*}{v}_{5, \mu}(\alpha) X_{5, \mu}(x ; \alpha),
\end{array}\right.
$$




$$
g(t, x ; \alpha)=\sum_{\mu=1}^{\infty} g_{1, \mu}(t ; \alpha) X_{1, \mu}(x ; \alpha)+\sum_{\mu=1}^{\infty} g_{5, \mu}(t ; \alpha) X_{5, \mu}(x ; \alpha)
$$

where the functions $X_{k, \mu}(x ; \alpha)$ are defined in Prop. 2.2 and the coefficients are calculated directly by integration

$$
\left\{\begin{aligned}
\stackrel{*}{v}_{k, \mu}(\alpha) & =\frac{1}{\left\|X_{k, \mu}\right\|^{2}} \int_{-1}^{+1} \stackrel{*}{v}(x ; \alpha) X_{k, \mu}(x ; \alpha) d x \\
\stackrel{v}{v}_{k, \mu}(\alpha) & =\frac{1}{\left\|X_{k, \mu}\right\|^{2}} \int_{-1}^{+1} \stackrel{* *}{v}(x ; \alpha) X_{k, \mu}(x ; \alpha) d x \\
g_{k, \mu}(t ; \alpha) & =\frac{1}{\left\|X_{k, \mu}\right\|^{2}} \int_{-1}^{+1} g(t, x ; \alpha) X_{k, \mu}(x ; \alpha) d x
\end{aligned}\right.
$$

Assuming that the ansatz for the required solution to the initial boundary value problem (2.23) to be as follows

$$
v(t, x ; \alpha)=\sum_{\mu=1}^{\infty} O_{1, \mu}(t ; \alpha) X_{1, \mu}(x ; \alpha)+\sum_{\mu=1}^{\infty} O_{5, \mu}(t ; \alpha) X_{5, \mu}(x ; \alpha),
$$

we obtain the Cauchy problems with respect to the desired coefficient functions of the ansatz

$$
\left\{\begin{array}{l}
O_{k, \mu}^{\prime \prime}(t ; \alpha)+\sigma_{k, \mu}^{2} O_{k, \mu}(t ; \alpha)=g_{k, \mu}(t ; \alpha), \quad t \in(0, T] \\
O_{k, \mu}^{\prime}(0 ; \alpha)=v_{k, \mu}^{* *}(\alpha) \\
O_{k, \mu}(0 ; \alpha)=v_{k, \mu}^{*}(\alpha)
\end{array}\right\} .
$$

The resulting expressions for the coefficients, after applying some trivial trigonometric manipulations, can be presented in the convolution form as follows

$$
\begin{aligned}
O_{k, \mu}(t ; \alpha) & =\stackrel{*}{v}_{k, \mu}(\alpha) \cos \left(\sigma_{k, \mu} t\right)+\sigma_{k, \mu}^{-1} \stackrel{* *}{v}_{k, \mu}(\alpha) \sin \left(\sigma_{k, \mu} t\right) \\
& +\sigma_{k, \mu}^{-1} \int_{0}^{t} g_{k, \mu}(z ; \alpha) \sin \left[\sigma_{k, \mu}(t-z)\right] \mathrm{d} z
\end{aligned}
$$

or, shortly, as

$$
\left\{\begin{aligned}
O_{k, \mu}(t ; \alpha) & =\stackrel{*}{v}_{k, \mu}(\alpha) \cos \left(\sigma_{k, \mu} t\right)+\sigma_{k, \mu}^{-1} \stackrel{* *}{v_{k, \mu}}(\alpha) \sin \left(\sigma_{k, \mu} t\right) \\
& +\sigma_{k, \mu}^{-1} g_{k, \mu}(t ; \alpha) \sin \left(\sigma_{k, \mu} t\right)
\end{aligned}\right.
$$


Finally, the representation (2.18) yields to the required unique bounded solution to the IBVP

$$
\left\{\begin{aligned}
u(t, x ; \alpha) & =\sum_{\mu=1}^{\infty} O_{1, \mu}(t ; \alpha) X_{1, \mu}(x ; \alpha) \\
& +\sum_{\mu=1}^{\infty} O_{5, \mu}(t ; \alpha) X_{5, \mu}(x ; \alpha) \\
& +\phi_{2}(x ; \alpha) h_{2}(t ; \alpha)+\phi_{1}(x ; \alpha) h_{1}(t ; \alpha) .
\end{aligned}\right.
$$

The above procedure can be readily interpreted in terms of decomposition of the functions $v(t, x ; \alpha), \stackrel{*}{v}(x ; \alpha), \stackrel{* *}{v}(x ; \alpha), w(t, x ; \alpha)$, and $g(t, x ; \alpha)$ into their even and odd parts, for example

$$
v(t, x ; \alpha)=v_{e}(t, x ; \alpha)+v_{o}(t, x ; \alpha), \quad x \in[-1,+1],
$$

where both parts are defined as follows

$$
\left\{\begin{array}{l}
2 v_{e}(t, x ; \alpha)=v(t,+x ; \alpha)+v(t,-x ; \alpha), \\
2 v_{o}(t, x ; \alpha)=v(t,+x ; \alpha)-v(t,-x ; \alpha),
\end{array}\right.
$$

leading to decomposition of the initial boundary value problem (2.23) into the derived problems

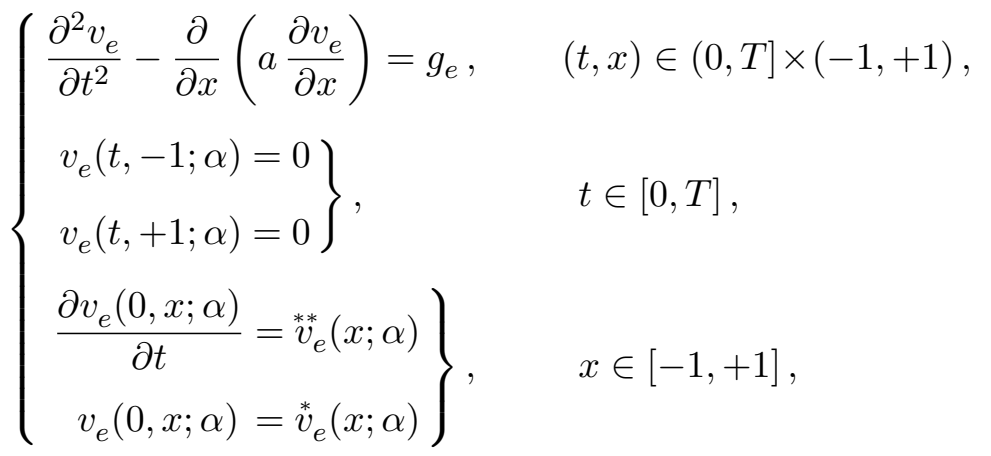

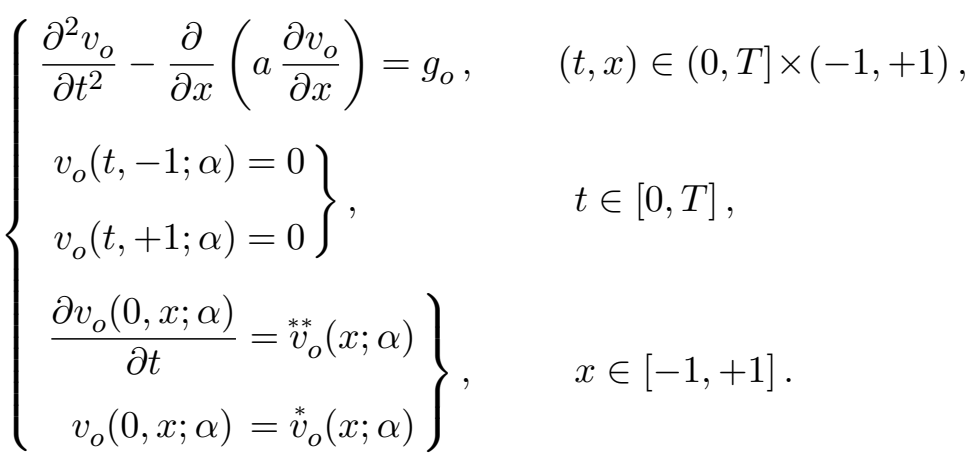


Applying SV to the above problems yields to the bounded solutions in the form of the following series

$$
\left\{\begin{array}{l}
v_{e}(t, x ; \alpha)=\sum_{\mu=1}^{\infty} O_{e, \mu}(t ; \alpha) X_{1, \mu}(x ; \alpha), \\
v_{o}(t, x ; \alpha)=\sum_{\mu=1}^{\infty} O_{o, \mu}(t ; \alpha) X_{5, \mu}(x ; \alpha),
\end{array}\right.
$$

where the coefficient functions $O_{e, \mu}(t ; \alpha)$ and $O_{o, \mu}(t ; \alpha)$ are evidently the solutions to respectively the same Cauchy problems (2.29). And eventually, using the representations (2.33), (2.32) and (2.18), the same unique bounded solution to the IBVP can be found again.

Calculating the flux of the obtained solution to the IBVP

$$
\left\{\begin{aligned}
-f(t, x ; \alpha) & =\sum_{\mu=1}^{\infty} O_{1, \mu}(t ; \alpha)\left[a(x ; \alpha) X_{1, \mu}^{\prime}(x ; \alpha)\right] \\
& +\sum_{\mu=1}^{\infty} O_{5, \mu}(t ; \alpha)\left[a(x ; \alpha) X_{5, \mu}^{\prime}(x ; \alpha)\right] \\
& +\psi_{2}(x ; \alpha) h_{2}(t ; \alpha)+\psi_{1}(x ; \alpha) h_{1}(t ; \alpha)
\end{aligned}\right.
$$

proves that the following condition holds

$$
f(t, 0-0 ; \alpha)=f(t, 0+0 ; \alpha), \quad t \in[0, T],
$$

due to: $a$ ) Prop. 2.2 and $b$ ) the regularity conditions (2.21) imposed on the blending functions $\phi_{1}(x ; \alpha)$ and $\phi_{2}(x ; \alpha)$ (or, shortly, due to continuous differentiability of the function $w(t, x ; \alpha)(2.19))$.

\subsection{Implementing $\mathrm{SV}$ to the $\mathrm{IBVP}_{1}$ and the $\mathrm{IBVP}_{2}$}

The required solutions to the $\mathrm{IBVP}_{j}$ in the case of strong degeneracy are assumed to have the following representation

$$
u_{j}(t, x ; \alpha)=v_{j}(t, x ; \alpha)+w_{j}(t, x ; \alpha),
$$

where: $a)$ the functions $v_{j}(t, x ; \alpha)$ are required; $\left.b\right)$ the functions $w_{j}(t, x ; \alpha)$ are given as follows

$$
w_{j}(t, x ; \alpha)=\phi_{j}(x ; \alpha) h_{j}(t ; \alpha)+\phi_{j+2}(x ; \alpha) h_{j+2}(t ; \alpha),
$$

$c)$ the smooth blending functions $\phi_{j}(x ; \alpha), \phi_{j+2}(x ; \alpha)$ satisfy the following boundary and regularity conditions, respectively:

$$
\begin{cases}\phi_{1}(+1 ; \alpha)=1, & \phi_{1}(0 ; \alpha)=0, \\ \phi_{3}(+1 ; \alpha)=0, & \phi_{3}(0 ; \alpha)=1,\end{cases}
$$




$$
\begin{gathered}
\begin{cases}\phi_{2}(-1 ; \alpha)=1, & \phi_{2}(0 ; \alpha)=0, \\
\phi_{4}(-1 ; \alpha)=0, & \phi_{4}(0 ; \alpha)=1,\end{cases} \\
\left\{\begin{array}{l}
\psi_{1}(x ; \alpha) \equiv \varphi_{1}^{\prime}(x ; \alpha)=\left[a(x ; \alpha) \phi_{1}^{\prime}(x ; \alpha)\right]^{\prime} \\
\psi_{3}(x ; \alpha) \equiv \varphi_{3}^{\prime}(x ; \alpha)=\left[a(x ; \alpha) \phi_{3}^{\prime}(x ; \alpha)\right]^{\prime} \quad \in \mathscr{C}[0,+1],
\end{array}\right. \\
\left\{\begin{array}{l}
\psi_{2}(x ; \alpha) \equiv \varphi_{2}^{\prime}(x ; \alpha)=\left[a(x ; \alpha) \phi_{2}^{\prime}(x ; \alpha)\right]^{\prime} \\
\psi_{4}(x ; \alpha) \equiv \varphi_{4}^{\prime}(x ; \alpha)=\left[a(x ; \alpha) \phi_{4}^{\prime}(x ; \alpha)\right]^{\prime}
\end{array}\right.
\end{gathered}
$$

d) $h_{j+2}(t ; \alpha)$ are the required corrections to $v_{j}(t, x ; \alpha)$ at the degeneracy segment.

Assuming that $h_{j+2}(0 ; \alpha)=\stackrel{*}{v}(0, x ; \alpha), h_{j+2}^{\prime}(0 ; \alpha)=\stackrel{* *}{v}(0, x ; \alpha)$ and combining $(2.36)-(2.39)$ yields to: $a)$ the initial conditions for $v_{j}(t, x ; \alpha)$

$$
\left\{\begin{aligned}
v_{j}(0, x ; \alpha) & =u_{j}(0, x ; \alpha)-w_{j}(0, x ; \alpha) \equiv v_{j}(x ; \alpha), \\
\frac{\partial v_{j}(0, x ; \alpha)}{\partial t} & =\frac{\partial u_{j}(0, x ; \alpha)}{\partial t}-\frac{\partial w_{j}(0, x ; \alpha)}{\partial t} \equiv v_{j}^{* *}(x ; \alpha),
\end{aligned}\right.
$$

and $b$ ) reformulation of the $\mathrm{IBVP}_{j}$ into the following auxiliary $\operatorname{IBVP}_{j}^{a}$ with respect to the functions $v_{j}(t, x ; \alpha)$

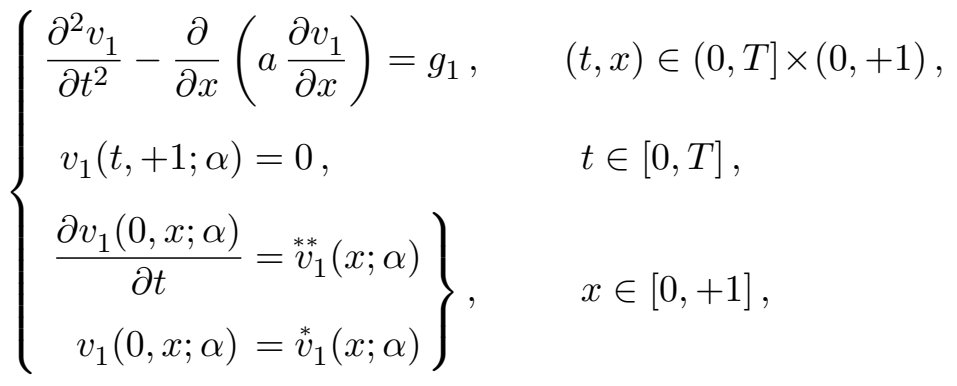

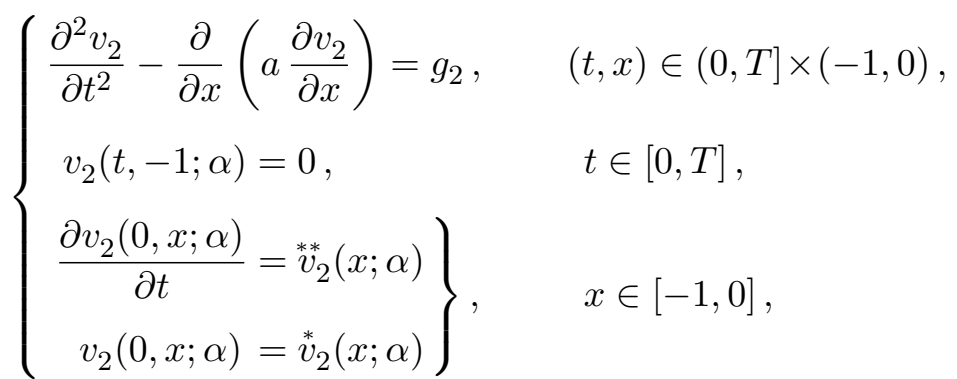

where the right-hand sides of the above degenerate wave equations

$$
g_{j}(t, x ; \alpha)=-\frac{\partial^{2} w_{j}(t, x ; \alpha)}{\partial t^{2}}+\frac{\partial}{\partial x}\left(a(x ; \alpha) \frac{\partial w_{j}(t, x ; \alpha)}{\partial x}\right),
$$

being expanded due to (2.37), read as follows

$$
\left\{\begin{aligned}
g_{j}(t, x ; \alpha)= & -\phi_{j}(x ; \alpha) h_{2}^{\prime \prime}(t ; \alpha)-\phi_{j+2}(x ; \alpha) h_{j+2}^{\prime \prime}(t ; \alpha) \\
& +\psi_{j}(x ; \alpha) h_{2}(t ; \alpha)+\psi_{j+2}(x ; \alpha) h_{j+2}(t ; \alpha) .
\end{aligned}\right.
$$


Then the initial functions (2.42) and the right-hand sides (2.45) are expanded into the series

$$
\begin{aligned}
& \left\{\begin{array}{l}
\stackrel{*}{v}_{j}(x ; \alpha)=\sum_{\mu=1}^{\infty} \stackrel{*}{v}_{j, \mu}(\alpha) Z_{1, \mu}(x ; \alpha), \\
\stackrel{* *}{v}_{j}(x ; \alpha)=\sum_{\mu=1}^{\infty} \stackrel{* *}{v}_{j, \mu}(\alpha) Z_{1, \mu}(x ; \alpha),
\end{array}\right. \\
& g_{j}(t, x ; \alpha)=\sum_{\mu=1}^{\infty} g_{j, \mu}(t ; \alpha) Z_{1, \mu}(x ; \alpha),
\end{aligned}
$$

where the coefficients are determined straightforwardly by integration. The expanded forms of the coefficients in (2.47) are

$$
\begin{aligned}
g_{j, \mu}(t ; \alpha) & =a_{j, \mu}(\alpha) h_{j}^{\prime \prime}(t ; \alpha)+c_{j, \mu}(\alpha) h_{j+2}^{\prime \prime}(t ; \alpha) \\
& +b_{j, \mu}(\alpha) h_{j}(t ; \alpha)+d_{j, \mu}(\alpha) h_{j+2}(t ; \alpha)
\end{aligned}
$$

where

$$
\begin{aligned}
& \left\{\begin{array}{l}
a_{1, \mu}(\alpha)=-\frac{1}{\left\|Z_{1, \mu}\right\|^{2}} \int_{0}^{+1} \phi_{1}(x ; \alpha) Z_{1, \mu}(x ; \alpha) d x, \\
b_{1, \mu}(\alpha)=+\frac{1}{\left\|Z_{1, \mu}\right\|^{2}} \int_{0}^{+1} \psi_{1}(x ; \alpha) Z_{1, \mu}(x ; \alpha) d x \\
c_{1, \mu}(\alpha)=-\frac{1}{\left\|Z_{1, \mu}\right\|^{2}} \int_{0}^{+1} \phi_{3}(x ; \alpha) Z_{1, \mu}(x ; \alpha) d x \\
d_{1, \mu}(\alpha)=+\frac{1}{\left\|Z_{1, \mu}\right\|^{2}} \int_{0}^{+1} \psi_{3}(x ; \alpha) Z_{1, \mu}(x ; \alpha) d x \\
b_{2, \mu}(\alpha)=+\frac{1}{\left\|Z_{1, \mu}\right\|^{2}} \int_{-1}^{0} \psi_{2}(x ; \alpha) Z_{1, \mu}(x ; \alpha) d x \\
a_{2, \mu}(\alpha)=-\frac{1}{\left\|Z_{1, \mu}\right\|^{2}} \int_{-1}^{0} \phi_{2}(x ; \alpha) Z_{1, \mu}(x ; \alpha) d x \\
c_{2, \mu}(\alpha)=-\frac{1}{\left\|Z_{1, \mu}\right\|^{2}} \int_{-1}^{0} \phi_{4}(x ; \alpha) Z_{1, \mu}(x ; \alpha) d x \\
d_{2, \mu}(\alpha)=+\frac{1}{\left\|Z_{1, \mu}\right\|^{2}} \int_{-1}^{0} \psi_{4}(x ; \alpha) Z_{1, \mu}(x ; \alpha) d x
\end{array}\right.
\end{aligned}
$$

And now substituting the ansatz for the solutions

$$
v_{j}(t, x ; \alpha)=\sum_{\mu=1}^{\infty} O_{j, \mu}(t ; \alpha) Z_{1, \mu}(x ; \alpha)
$$


into the $\operatorname{IBVP}_{j}^{a}$ obtains the Cauchy problems for the coefficient functions

$$
\left\{\begin{array}{l}
O_{j, \mu}^{\prime \prime}(t ; \alpha)+\sigma_{1, \mu}^{2} O_{j, \mu}(t ; \alpha)=g_{j, \mu}(t ; \alpha), \quad t \in(0, T] \\
O_{j, \mu}^{\prime}(0 ; \alpha)=v_{j, \mu}^{* *}(\alpha) \\
O_{j, \mu}(0 ; \alpha)=v_{j, \mu}^{*}(\alpha)
\end{array}\right\} .
$$

The resulting expressions for the coefficients can be readily presented in the convolution form as follows

$$
\left\{\begin{aligned}
O_{j, \mu}(t ; \alpha) & =\stackrel{*}{v}_{j, \mu}(\alpha) \cos \left(\sigma_{1, \mu} t\right)+\sigma_{k, \mu}^{-1} \stackrel{* *}{v}_{j, \mu}(\alpha) \sin \left(\sigma_{1, \mu} t\right) \\
& +\sigma_{1, \mu}^{-1} g_{j, \mu}(t ; \alpha) \sin \left(\sigma_{1, \mu} t\right)
\end{aligned}\right.
$$

Finally, the representation (2.36) obtains the required solutions to the $\mathrm{IBVP}_{j}$

$$
\begin{aligned}
& \left\{\begin{aligned}
u_{1}(t, x ; \alpha) & =\sum_{\mu=1}^{\infty} O_{1, \mu}(t ; \alpha) Z_{1, \mu}(x ; \alpha) \\
& +\phi_{1}(x ; \alpha) h_{1}(t ; \alpha)+\phi_{3}(x ; \alpha) h_{3}(t ; \alpha),
\end{aligned}\right. \\
& \left\{\begin{aligned}
u_{2}(t, x ; \alpha) & =\sum_{\mu=1}^{\infty} O_{2, \mu}(t ; \alpha) Z_{1, \mu}(x ; \alpha) \\
& +\phi_{2}(x ; \alpha) h_{2}(t ; \alpha)+\phi_{4}(x ; \alpha) h_{4}(t ; \alpha) .
\end{aligned}\right.
\end{aligned}
$$

Calculating the fluxes of the obtained solutions $u_{j}(t, x ; \alpha)$

$$
\begin{aligned}
& \left\{\begin{aligned}
-f_{1}(t, x ; \alpha) & =\sum_{\mu=1}^{\infty} O_{1, \mu}(t ; \alpha)\left[a(x ; \alpha) Z_{1, \mu}^{\prime}(x ; \alpha)\right] \\
& +\psi_{1}(x ; \alpha) h_{1}(t ; \alpha)+\psi_{3}(x ; \alpha) h_{3}(t ; \alpha),
\end{aligned}\right. \\
& \left\{\begin{aligned}
-f_{2}(t, x ; \alpha) & =\sum_{\mu=1}^{\infty} O_{2, \mu}(t ; \alpha)\left[a(x ; \alpha) Z_{1, \mu}^{\prime}(x ; \alpha)\right] \\
& +\psi_{2}(x ; \alpha) h_{2}(t ; \alpha)+\psi_{4}(x ; \alpha) h_{4}(t ; \alpha),
\end{aligned}\right.
\end{aligned}
$$

proves that the following condition holds

$$
f_{2}(t, 0-0 ; \alpha)=f_{1}(t, 0+0 ; \alpha)=0, \quad t \in[0, T],
$$

yet before matching the solutions, due to: $a$ ) Prop. 2.1 and $b$ ) the regularity conditions (2.40) and (2.41) imposed on the blending functions $\phi_{j}(x ; \alpha)$ and $\phi_{j+2}(x ; \alpha)$ (or, shortly, due to continuous differentiability of the functions $w_{j}(t, x ; \alpha)(2.37)$ ). 


\subsection{Matching the Solutions to the $\operatorname{IBVP}_{1}$ and the $\mathrm{IBVP}_{2}$}

To implement matching the obtained one-sided solutions $u_{1}(t, x ; \alpha)(2.54)$ and $u_{2}(t, x ; \alpha)(2.55)$, we will follow the procedure:

1) substitute the above solutions into the matching condition (2.17), as follows

$$
\begin{aligned}
& \sum_{\mu=1}^{\infty} O_{2, \mu}(t ; \alpha) Z_{1, \mu}(0 ; \alpha)+\phi_{2}(0 ; \alpha) h_{2}(t ; \alpha)+\phi_{4}(0 ; \alpha) h_{4}(t ; \alpha) \\
= & \sum_{\mu=1}^{\infty} O_{1, \mu}(t ; \alpha) Z_{1, \mu}(0 ; \alpha)+\phi_{1}(0 ; \alpha) h_{1}(t ; \alpha)+\phi_{3}(0 ; \alpha) h_{3}(t ; \alpha) ;
\end{aligned}
$$

2) replace the values $Z_{1, \mu}(0 ; \alpha)$ with the pre-derived formula $(2.12)$

$$
Z_{1, \mu}(0 ; \alpha)=\left(\frac{s_{1, \mu}}{2}\right)^{-\varrho} \frac{1}{\Gamma(1-\varrho)}=\frac{\theta^{+\varrho} \sigma_{1, \mu}^{-\varrho}}{\Gamma(1-\varrho)} \equiv C_{\varrho} \sigma_{1, \mu}^{-\varrho}
$$

$3)$ account for the boundary conditions (2.38) and (2.39) imposed on the blending functions $\phi_{j}(x ; \alpha)$ and $\phi_{j+2}(x ; \alpha)$, to obtain the following linear integro-differential equation of convolution type with respect to $h_{3}(t ; \alpha)$ and $h_{4}(t ; \alpha)$

$$
C_{\varrho} \sum_{\mu=1}^{\infty} \sigma_{1, \mu}^{-\varrho} O_{2, \mu}(t ; \alpha)+h_{4}(t ; \alpha)=C_{\varrho} \sum_{\mu=1}^{\infty} \sigma_{1, \mu}^{-\varrho} O_{1, \mu}(t ; \alpha)+h_{3}(t ; \alpha) .
$$

The above representation of the matching condition (2.17) can be rewritten in the expanded form

$$
\left\{\begin{aligned}
& a_{2}(t ; \alpha) * h_{2}^{\prime \prime}(t ; \alpha)+b_{2}(t ; \alpha) * h_{2}(t ; \alpha)+\stackrel{v}{v}_{2}(t ; \alpha)+\stackrel{v}{v}_{2}^{* *}(t ; \alpha) \\
+ & c_{2}(t ; \alpha) * h_{4}^{\prime \prime}(t ; \alpha)+d_{2}(t ; \alpha) * h_{4}(t ; \alpha)+h_{4}(t ; \alpha) \\
= & a_{1}(t ; \alpha) * h_{1}^{\prime \prime}(t ; \alpha)+b_{1}(t ; \alpha) * h_{1}(t ; \alpha)+v_{v}^{*}(t ; \alpha)+\stackrel{*}{v}_{1}^{* *}(t ; \alpha) \\
+ & c_{1}(t ; \alpha) * h_{3}^{\prime \prime}(t ; \alpha)+d_{1}(t ; \alpha) * h_{3}(t ; \alpha)+h_{3}(t ; \alpha)
\end{aligned}\right.
$$

where the coefficient functions are defined by the following series

$$
\left\{\begin{array}{l}
\stackrel{*}{v}_{j}(t ; \alpha)=C_{\varrho} \sum_{\mu=1}^{\infty} \sigma_{1, \mu}^{-\varrho} \quad \stackrel{*}{v}_{j, \mu}(\alpha) \cos \left(\sigma_{1, \mu} t\right) \\
y_{j}(t ; \alpha)=C_{\varrho} \sum_{\mu=1}^{\infty} \sigma_{1, \mu}^{-\varrho-1} y_{j, \mu}(\alpha) \sin \left(\sigma_{1, \mu} t\right)
\end{array}\right.
$$

and one should substitute symbols ' $\stackrel{* *}{v}$, ' $a$ ', ' $b$ ', ' $c$ ', and ' $d$ ' instead of ' $y$ ' in (2.60). 


\section{The Laplace Transformation}

\subsection{Finding the Images}

To solve the integro-differential equation (2.59) of convolution type, we apply the Laplace transformation [3], producing for a function $f(t), t \in[0, \infty)$, its transform as follows

$$
F(\tau)=\mathfrak{L}[f(t)]:=\int_{0}^{\infty} f(t) \mathrm{e}^{-\tau t} d t, \quad \tau=\xi+i \eta \in \mathbb{C},
$$

provided the original function $f(t)$ satisfies the known sufficient conditions for the image function $F(\tau)$ to exist.

When applying the Laplace transformation we use:

1) the convolution theorem

$$
\mathfrak{L}[p(t) * q(t)]=\mathfrak{L}[p(t)] \cdot \mathfrak{L}[q(t)]=P(\tau) \cdot Q(\tau),
$$

where the symbol 'middle dot' between the two images is used, where it is needed, for reminding about the origin of their multiplication;

2 ) the transforms of the control functions $h_{j}(t ; \alpha)$ and their second derivatives, accounting for the given initial conditions

$$
\begin{aligned}
& \mathfrak{L}\left[h_{j}(t ; \alpha)\right]=H_{j}(\tau ; \alpha), \\
& \mathfrak{L}\left[h_{j}^{\prime \prime}(t ; \alpha)\right]=H_{j}(\tau ; \alpha) \tau^{2}-h_{j}(0 ; \alpha) \tau-h_{j}^{\prime}(0 ; \alpha),
\end{aligned}
$$

3) the transforms of the required functions $h_{3}(t ; \alpha), h_{4}(t ; \alpha)$ and their second derivatives, accounting for the prescribed initial conditions

$$
\begin{aligned}
\mathfrak{L}\left[h_{j+2}(t ; \alpha)\right] & =H_{j+2}(\tau ; \alpha), \\
\mathfrak{L}\left[h_{j+2}^{\prime \prime}(t ; \alpha)\right] & =H_{j+2}(\tau ; \alpha) \tau^{2}-h_{j+2}(0 ; \alpha) \tau-h_{j+2}^{\prime}(0 ; \alpha),
\end{aligned}
$$

4) the transforms of the trigonometric sine and cosine functions

$$
\left\{\begin{array}{l}
\mathfrak{L}\left[\sin \left(\sigma_{1, \mu} t\right)\right]=\frac{\sigma_{1, \mu}}{\tau^{2}+\sigma_{1, \mu}^{2}} \equiv S_{\mu}(\tau ; \alpha), \\
\mathfrak{L}\left[\cos \left(\sigma_{1, \mu} t\right)\right]=\frac{\tau}{\tau^{2}+\sigma_{1, \mu}^{2}} \equiv C_{\mu}(\tau ; \alpha),
\end{array}\right.
$$

Then, the Laplace transformation applied to the equation (2.59) yields evidently to its image as follows

$$
\left\{\begin{array}{r}
{\left[1+Q_{2}(\tau ; \alpha)\right] \cdot H_{4}(\tau ; \alpha)+R_{2}(\tau ; \alpha)} \\
=\left[1+Q_{1}(\tau ; \alpha)\right] \cdot H_{3}(\tau ; \alpha)+R_{1}(\tau ; \alpha),
\end{array}\right.
$$


where

$$
\begin{aligned}
& R_{j}(\tau ; \alpha)=P_{j}(\tau ; \alpha) \cdot H_{j}(\tau ; \alpha)+V_{j}(\tau ; \alpha)-K_{j}(\tau ; \alpha)-N_{j}(\tau ; \alpha), \\
& \left\{\begin{aligned}
P_{j}(\tau ; \alpha) & =A_{j}(\tau ; \alpha) \tau^{2}+B_{j}(\tau ; \alpha) \\
& =C_{\varrho} \sum_{\mu=1}^{\infty} \sigma_{\varrho, \mu}^{-\varrho} \frac{a_{j, \mu}(\alpha) \tau^{2}+b_{j, \mu}(\alpha)}{\tau^{2}+\sigma_{1, \mu}^{2}},
\end{aligned}\right. \\
& \left\{\begin{aligned}
Q_{j}(\tau ; \alpha) & =C_{j}(\tau ; \alpha) \tau^{2}+D_{j}(\tau ; \alpha) \\
& =C_{\varrho} \sum_{\mu=1}^{\infty} \sigma_{\varrho, \mu}^{-\varrho} \frac{c_{j, \mu}(\alpha) \tau^{2}+d_{j, \mu}(\alpha)}{\tau^{2}+\sigma_{1, \mu}^{2}},
\end{aligned}\right. \\
& \left\{\begin{aligned}
V_{j}(\tau ; \alpha) & =\stackrel{*}{V}_{j}(\tau ; \alpha)+\stackrel{* *}{V}_{j}(\tau ; \alpha) \\
& =C_{\varrho} \sum_{\mu=1}^{\infty} \sigma_{\varrho, \mu}^{-\varrho} \frac{\stackrel{*}{v}_{j, \mu}(\alpha) \tau+\stackrel{*}{v}_{j, \mu}(\alpha)}{\tau^{2}+\sigma_{1, \mu}^{2}}
\end{aligned}\right. \\
& \left\{\begin{aligned}
K_{j}(\tau ; \alpha) & =\stackrel{*}{K}_{j}(\tau ; \alpha) h_{j}(0 ; \alpha)+\stackrel{* *}{K}_{j}(\tau ; \alpha) h_{j}^{\prime}(0 ; \alpha) \\
& =C_{\varrho} \sum_{\mu=1}^{\infty} \sigma_{\varrho, \mu}^{-\varrho} a_{j, \mu}(\alpha) \frac{h_{j}(0 ; \alpha) \tau+h_{j}^{\prime}(0 ; \alpha)}{\tau^{2}+\sigma_{1, \mu}^{2}},
\end{aligned}\right. \\
& \left\{\begin{aligned}
N_{j}(\tau ; \alpha) & =\stackrel{*}{N}_{j}(\tau ; \alpha) h_{j+2}(0 ; \alpha)+\stackrel{* *}{N}{ }_{j}(\tau ; \alpha) h_{j+2}^{\prime}(0 ; \alpha) \\
& =C_{\varrho} \sum_{\mu=1}^{\infty} \sigma_{\varrho, \mu}^{-\varrho} c_{j, \mu}(\alpha) \frac{h_{j+2}(0 ; \alpha) \tau+h_{j+2}^{\prime}(0 ; \alpha)}{\tau^{2}+\sigma_{1, \mu}^{2}}
\end{aligned}\right.
\end{aligned}
$$

the functions $A_{j}(\tau ; \alpha), B_{j}(\tau ; \alpha), C_{j}(\tau ; \alpha), D_{j}(\tau ; \alpha), \stackrel{*}{V}_{j}(\tau ; \alpha)$, and $\stackrel{* *}{V}(\tau ; \alpha)$ being the images of the functions $a_{j}(t ; \alpha), b_{j}(t ; \alpha), c_{j}(t ; \alpha), d_{j}(t ; \alpha), v_{j}^{*}(t ; \alpha)$, and $\stackrel{*}{v}_{j}^{* *}(t ; \alpha)$.

Assuming that $\phi_{1,4}(x ; \alpha)=\phi_{1,3}(-x ; \alpha)$ (see Section 2 at p. 24), we easily conclude that: 1$)$ the equality $Q_{1}(\tau ; \alpha)=Q_{2}(\tau ; \alpha)$ holds; 2$)$ the image (3.4) of the matching condition $(2.59)$ reduces to

$$
\left[1+Q_{1}(\tau ; \alpha)\right] \cdot \Delta H(\tau ; \alpha)=R_{2}(\tau ; \alpha)-R_{1}(\tau ; \alpha),
$$

where $\Delta H(\tau ; \alpha) \equiv H_{3}(\tau ; \alpha)-H_{4}(\tau ; \alpha)$, or after dividing both sides of (3.6) by $\left[1+Q_{1}(\tau ; \alpha)\right]$, to the formula

$$
\Delta H(\tau ; \alpha)=\frac{R_{2}(\tau ; \alpha)-R_{1}(\tau ; \alpha)}{1+Q_{1}(\tau ; \alpha)}
$$




\subsection{Finding the Original Functions}

We start from estimating applicability of some known approaches to invert the formula (3.7) and find the original function $h(t ; \alpha)=h_{3}(t ; \alpha)-h_{4}(t ; \alpha)$.

a) We could expect that rewriting the formula (3.7) as follows

$$
\Delta H(\tau ; \alpha)=\left[1+Q_{1}(\tau ; \alpha)\right]^{-1} \cdot\left[R_{2}(\tau ; \alpha)-R_{1}(\tau ; \alpha)\right]
$$

makes it possible to invoke the convolution theorem (3.2) and find the function $h(t ; \alpha)$ provided both multipliers in (3.8) are the images.

To estimate this approach to be useful, we take into account that for any original function $f(t)$ its transform $F(\tau)$ is necessarily [3]: 1) analytic in the right half of the $\tau$-plane: $\Re \tau>\xi^{*}>0$, where $\xi^{*}$ is some proper real value; 2$)$ vanishing when $\Re \tau \rightarrow+\infty$.

Proposition 3.1. The functions $R_{1}(\tau ; \alpha), R_{2}(\tau ; \alpha)$ (3.5) are transforms, whereas the function $\left[1+Q_{1}(\tau ; \alpha)\right]^{-1}$ is not a transform.

Proof. Let's turn to the expressions (3.5). The functions $P_{j}(\tau ; \alpha)$ are analytic in the whole $\tau$-plane except for the simple poles $\tau_{\mu}^{\mp}=\mp i \sigma_{1, \mu}[5]$ and both do not vanish when $\Re \tau \rightarrow+\infty$. Nevertheless, both products $P_{j}(\tau ; \alpha) \cdot H_{j}(\tau ; \alpha)$ vanish when $\Re \tau \rightarrow+\infty$, since both multipliers $H_{j}(\tau ; \alpha)$ are the transforms of the control functions $h_{j}(t ; \alpha)$. From this we conclude that the above products are transforms as well. Then, we notice that the functions $\stackrel{*}{V_{j}}(\tau ; \alpha)$ and $\stackrel{* *}{V}(\tau ; \alpha), \stackrel{*}{K_{j}}(\tau ; \alpha)$ and $\stackrel{* *}{K}_{j}(\tau ; \alpha), \stackrel{*}{N}_{j}(\tau ; \alpha)$ and $\stackrel{* *}{N}_{j}(\tau ; \alpha)$ are themselves the transforms, and this completes the proof of the first part of the proposition.

The function $Q_{1}(\tau ; \alpha)$ has the same properties as both functions $P_{j}(\tau ; \alpha)$ have, therefore the function $\left[1+Q_{1}(\tau ; \alpha)\right]^{-1}$ is not a transform, and this completes the proof of the second part of the proposition.

Although the first approach turnes out to be unsuccessful, nevertheless it follows from Proposition 3.1 that the right-hand side of the formula (3.7) is indeed the transform of the required function $h(t ; \alpha)$.

b) The next approach is to invert the right-hand side of the formula (3.7) directly. Indeed, let the Laplace transform $F(\tau)$ for an original function $f(t)$ be given, then applying the inverse Laplace transformation [3], known also as the Bromwich integral, yields to the required original function

$$
f(t)=\mathfrak{L}^{-1}[F(\tau)]=\frac{1}{2 \pi i} \int_{\xi^{*}-i \infty}^{\xi^{*}+i \infty} F(\tau) \mathrm{e}^{+t \tau} d \tau,
$$

where $\Re \tau=\xi^{*}$ is a vertical straight line lying to the right of all the singularities of $F(\tau)$ (see Fig. 3.1, a).

Practically, calculating the Bromwich integral is performed using the Cauchy residue theorem [5], but this approach implies that the singularities of the integrand 

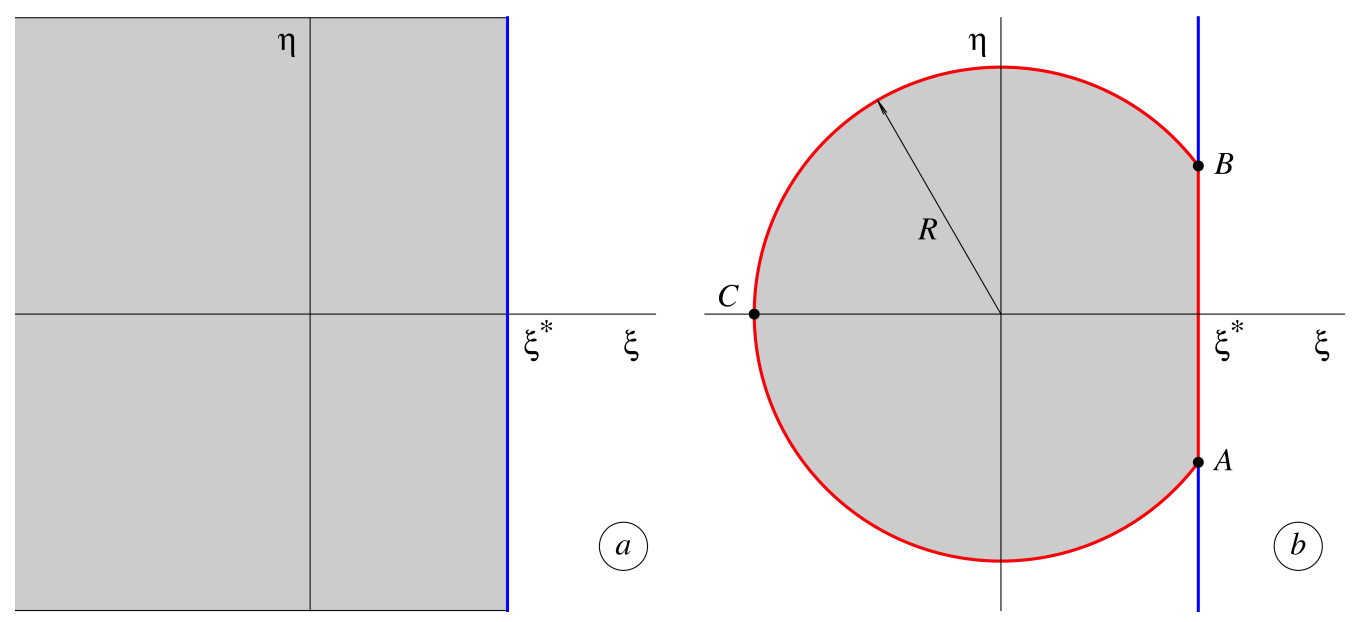

Fig. 3.1. All the singularities of the integrand of the Bromwich integral (3.9) lie in the half-plane (gray color) to the right of the path of integration $\tau=\xi^{*}+i \eta, \xi^{*}=$ const, $\eta \in(-\infty,+\infty)$ (dark blue), or the Bromwich line (a); the oriented Bromwich countour ABCA (light red), consists of the segment $A B$ of the Bromwich line and the arc $B C A$ of the circle of radius $R$ centered at the origin; to apply the Cauchy residue theorem, the integrand must vanish at $B C A$ when $R \rightarrow \infty$ and all the singularities of the integrand must lie inside $A B C A(b)$

are isolated and known (see Fig. 3.1, b). The functions $P_{j}(\tau ; \alpha)$ and $Q_{1}(\tau ; \alpha)$ have the same poles being removable singularities of the integrand and having no impact on calculating the integral; whereas finding all zeros of the function $\left[1+Q_{1}(\tau ; \alpha)\right]$ generally implies some proper approximation [4] of the latter and results in a huge bulk of the computational work. Therefore, we do not reject calculating the Bromwich integral at all, but postpone applying this approach for a while.

c) To implement the third approach, we:

1) recombine the terms in the series (3.5)

$$
\begin{aligned}
& \left\{\begin{aligned}
K_{j}(\tau ; \alpha) & =C_{\varrho}\left(\sum_{\mu=1}^{\infty} \sigma_{1, \mu}^{-\varrho} a_{j, \mu}(\alpha) C_{\mu}(\tau ; \alpha)\right) h_{j}(0 ; \alpha) \\
& +C_{\varrho}\left(\sum_{\mu=1}^{\infty} \sigma_{1, \mu}^{-\varrho-1} a_{j, \mu}(\alpha) S_{\mu}(\tau ; \alpha)\right) h_{j}^{\prime}(0 ; \alpha),
\end{aligned}\right. \\
& \left\{\begin{aligned}
N_{j}(\tau ; \alpha) & =C_{\varrho}\left(\sum_{\mu=1}^{\infty} \sigma_{1, \mu}^{-\varrho} c_{j, \mu}(\alpha) C_{\mu}(\tau ; \alpha)\right) h_{j+2}(0 ; \alpha) \\
& +C_{\varrho}\left(\sum_{\mu=1}^{\infty} \sigma_{1, \mu}^{-\varrho-1} c_{j, \mu}(\alpha) S_{\mu}(\tau ; \alpha)\right) h_{j+2}^{\prime}(0 ; \alpha),
\end{aligned}\right.
\end{aligned}
$$




$$
\begin{aligned}
& \left\{\begin{aligned}
P_{j}(\tau ; \alpha)-\overbrace{C_{\varrho} \sum_{\mu=1}^{\infty} \sigma_{1, \mu}^{-\varrho} a_{j, \mu}(\alpha)}^{\Psi_{j}(\alpha)} & -C_{\varrho} \sum_{\mu=1}^{\infty} \sigma_{1, \mu}^{-\varrho+1} a_{j, \mu}(\alpha) S_{\mu}(\tau ; \alpha) \\
& +C_{\varrho} \sum_{\mu=1}^{\infty} \sigma_{1, \mu}^{-\varrho-1} b_{j, \mu}(\alpha) S_{\mu}(\tau ; \alpha),
\end{aligned}\right. \\
& \left\{\begin{aligned}
\overbrace{C_{\varrho} \sum_{\mu=1}^{\infty} \sigma_{1, \mu}^{-\varrho} c_{j, \mu}(\alpha)}^{\Omega_{1}(\alpha)}-Q_{1}(\tau ; \alpha)= & +C_{\varrho} \sum_{\mu=1}^{\infty} \sigma_{1, \mu}^{-\varrho+1} c_{j, \mu}(\alpha) S_{\mu}(\tau ; \alpha) \\
& -C_{\varrho} \sum_{\mu=1}^{\infty} \sigma_{1, \mu}^{-\varrho-1} d_{j, \mu}(\alpha) S_{\mu}(\tau ; \alpha) \equiv \hat{Q}_{1}(\tau ; \alpha),
\end{aligned}\right.
\end{aligned}
$$

and easily find the respective original functions

$$
\begin{aligned}
& \left\{\begin{aligned}
p_{j}(t ; \alpha)= & -C_{\varrho} \sum_{\mu=1}^{\infty} \sigma_{1, \mu}^{-\varrho+1} a_{j, \mu}(\alpha) \sin \left(\sigma_{1, \mu} t\right) \\
& +C_{\varrho} \sum_{\mu=1}^{\infty} \sigma_{1, \mu}^{-\varrho-1} b_{j, \mu}(\alpha) \sin \left(\sigma_{1, \mu} t\right)+\Psi_{j}(\alpha) \delta(t),
\end{aligned}\right. \\
& \left\{\begin{aligned}
\hat{q}_{1}(t ; \alpha)= & +C_{\varrho} \sum_{\mu=1}^{\infty} \sigma_{1, \mu}^{-\varrho+1} c_{j, \mu}(\alpha) \sin \left(\sigma_{1, \mu} t\right) \\
& -C_{\varrho} \sum_{\mu=1}^{\infty} \sigma_{1, \mu}^{-\varrho-1} d_{j, \mu}(\alpha) \sin \left(\sigma_{1, \mu} t\right),
\end{aligned}\right.
\end{aligned}
$$

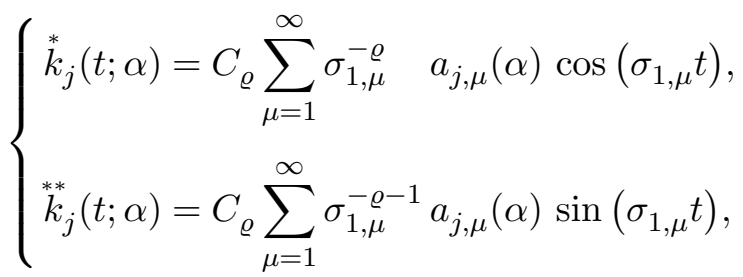

$$
\begin{aligned}
& \left\{\begin{array}{l}
\stackrel{*}{n}_{j}(t ; \alpha)=C_{\varrho} \sum_{\mu=1}^{\infty} \sigma_{1, \mu}^{-\varrho} \quad c_{j, \mu}(\alpha) \cos \left(\sigma_{1, \mu} t\right), \\
\stackrel{* *}{n}_{j}(t ; \alpha)=C_{\varrho} \sum_{\mu=1}^{\infty} \sigma_{1, \mu}^{-\varrho} c_{j, \mu}(\alpha) \sin \left(\sigma_{1, \mu} t\right),
\end{array}\right.
\end{aligned}
$$

where $\delta(t)$ is the Dirac delta function; 
2) represent the denominator of the formula (3.7) as follows

$$
\begin{aligned}
1+Q_{1}(\tau ; \alpha) & =1+\Omega_{1}(\alpha)-\hat{Q}_{1}(\tau ; \alpha) \equiv C_{\alpha}-\hat{Q}_{1}(\tau ; \alpha) \\
& =C_{\alpha}\left(1-C_{\alpha}^{-1} \hat{Q}_{1}(\tau ; \alpha)\right) \equiv C_{\alpha}\left(1-\bar{Q}_{1}(\tau ; \alpha)\right) ;
\end{aligned}
$$

3) rewrite the formula (3.7) as is usually done when solving integral equations of convolution type

$$
\Delta H(\tau ; \alpha)=C_{\alpha}^{-1}\left(1+\frac{\bar{Q}_{1}(\tau ; \alpha)}{1-\bar{Q}_{1}(\tau ; \alpha)}\right) \cdot\left[R_{2}(\tau ; \alpha)-R_{1}(\tau ; \alpha)\right]
$$

4) expand the 'fractional' term in (3.14) in the following power series

$$
\frac{\bar{Q}_{1}(\tau ; \alpha)}{1-\bar{Q}_{1}(\tau ; \alpha)}=\bar{Q}_{1}(\tau ; \alpha)+\sum_{\gamma=2}^{\infty}\left[\bar{Q}_{1}(\tau ; \alpha)\right]^{\gamma},
$$

provided that $\left|\bar{Q}_{1}(\tau ; \alpha)\right|<1$ in a proper right half-plane of the $\tau$-plane [3];

$5)$ invert the above power series in the form of the Neumann series [3]

$$
\mathfrak{L}^{-1}\left[\frac{\bar{Q}_{1}(\tau ; \alpha)}{1-\bar{Q}_{1}(\tau ; \alpha)}\right]=\bar{q}_{1}(t ; \alpha)+\sum_{\gamma=2}^{\infty}\left[\bar{q}_{1}(t ; \alpha)\right]^{* \gamma} \equiv \Phi(t ; \alpha),
$$

or the sum of iterated kernels, where $\bar{q}_{1}(t ; \alpha)=C_{\alpha}^{-1} \hat{q}_{1}(t ; \alpha)(3.11)$;

$6)$ invert the terms in the brackets in (3.14)

$$
\left\{\begin{aligned}
r_{j}(t ; \alpha) & =p_{j}(t ; \alpha) * h_{j}(t)+\stackrel{*}{v}_{j}(t ; \alpha)+\stackrel{* *}{v}_{j}(t ; \alpha) \\
& -\stackrel{*}{k}_{j}(t ; \alpha) h_{j}(0 ; \alpha)-\stackrel{*}{n}_{j}(t ; \alpha) h_{j+2}(0 ; \alpha) \\
& -\stackrel{* *}{k}_{j}(t ; \alpha) h_{j}^{\prime}(0 ; \alpha)-\stackrel{* *}{n}_{j}(t ; \alpha) h_{j+2}^{\prime}(0 ; \alpha) ;
\end{aligned}\right.
$$

7) finally, invert the formula (3.7) by invoking the convolution theorem (3.2)

$$
\left\{\begin{array}{rrr}
C_{\alpha}\left(h_{3}(t ; \alpha)-h_{4}(t ; \alpha)\right) & = & {\left[r_{2}(t ; \alpha)-r_{1}(t ; \alpha)\right]} \\
& +\Phi(t ; \alpha) *\left[r_{2}(t ; \alpha)-r_{1}(t ; \alpha)\right] .
\end{array}\right.
$$

\section{Conclusions}

1. In the current study we have applied the previously obtained [2] one-sided solutions $u_{1, j}(t, x ; \alpha), u_{5, j}(t, x ; \alpha)(1.3),(1.4)$ to the degenerate wave equation as the building blocks of procedures for finding bounded solutions to the IBVP (1.1), posed in the space-time rectangle $[0, T] \times[-1,+1]$, in the cases of weak $(\alpha \in(0,1))$ and strong $(\alpha \in(1,2))$ degeneracy. 
2. In the case of weak degeneracy the bounded unique solution (2.31) to the IBVP

$$
\left\{\begin{aligned}
u(t, x ; \alpha) & =\sum_{\mu=1}^{\infty} O_{1, \mu}(t ; \alpha) X_{1, \mu}(x ; \alpha) \\
& +\sum_{\mu=1}^{\infty} O_{5, \mu}(t ; \alpha) X_{5, \mu}(x ; \alpha) \\
& +\phi_{2}(x ; \alpha) h_{2}(t ; \alpha)+\phi_{1}(x ; \alpha) h_{1}(t ; \alpha)
\end{aligned}\right.
$$

has been obtained in the space-time rectangle $[0, T] \times[-1,+1]$, using the method of SV based on the eigenfunctions $X_{1, \mu}(x ; \alpha)$ and $X_{5, \mu}(x ; \alpha)$, defined in Prop. 2.2 at p. 6 . The solution (4.1) satisfies the following two continuity conditions

$$
\left\{\begin{array}{l}
u(t, 0-0 ; \alpha)=u(t, 0+0 ; \alpha), \\
f(t, 0-0 ; \alpha)=f(t, 0+0 ; \alpha),
\end{array} \quad t \in[0, T],\right.
$$

at the degeneracy segment, where $f(t, x ; \alpha)$ is the flux of the solution.

3 . In the case of strong degeneracy a family of bounded non-unique solutions to the IBVP have been obtained in the space-time rectangle $[0, T] \times[-1,+1]$, applying the following procedure:

a) two families of bounded solutions (2.54), (2.55)

$$
\begin{aligned}
& \left\{\begin{aligned}
u_{1}(t, x ; \alpha) & =\sum_{\mu=1}^{\infty} O_{1, \mu}(t ; \alpha) Z_{1, \mu}(x ; \alpha) \\
& +\phi_{1}(x ; \alpha) h_{1}(t ; \alpha)+\phi_{3}(x ; \alpha) h_{3}(t ; \alpha),
\end{aligned}\right. \\
& \left\{\begin{aligned}
u_{2}(t, x ; \alpha) & =\sum_{\mu=1}^{\infty} O_{2, \mu}(t ; \alpha) Z_{1, \mu}(x ; \alpha) \\
& +\phi_{2}(x ; \alpha) h_{2}(t ; \alpha)+\phi_{4}(x ; \alpha) h_{4}(t ; \alpha) .
\end{aligned}\right.
\end{aligned}
$$

to the derived $\operatorname{IBVP}_{1}(2.14)$ and $\operatorname{IBVP}_{2}(2.15)$, posed in the 'right' $[0, T] \times[0,+1]$ and the 'left' $[0, T] \times[-1,0]$ space-time rectangles, are obtained, using the method of SV based on the eigenfunctions $Z_{1, \mu}(x ; \alpha)$, defined in Prop. 2.1 at p. 4 ;

$b$ ) the solutions of both families, depending on undetermined functions $h_{3}(t ; \alpha)$ and $h_{4}(t ; \alpha)$ and satisfying the only continuity condition for their fluxes

$$
f_{2}(t, 0-0 ; \alpha)=f_{1}(t, 0+0 ; \alpha), \quad t \in[0, T],
$$

are then matched to implement the other continuity condition

$$
u_{2}(t, 0-0 ; \alpha)=u_{1}(t, 0+0 ; \alpha), \quad t \in[0, T],
$$

nevertheless, the resulting matched family still retains one undetermined function. 


\section{Appendix. Calculating the Coefficients $a, b, c$, and $d$}

In this section the method of calculating the coefficients (2.49), (2.50) is presented. We take for $\phi_{k, j}(x ; \alpha)$ and $\phi_{k, j+2}(x ; \alpha)$ the following power functions

$$
\left\{\begin{aligned}
\phi_{j}(x ; \alpha) & =|x|^{\omega_{j}} \\
\phi_{j+2}(x ; \alpha) & =1-|x|^{\omega_{j+2}}
\end{aligned}\right.
$$

where the undetermined exponents $\omega_{k, j}$ and $\omega_{k, j+2}$ should be adjusted to the parameter $\alpha$ in a special way. To impose the proper constraint on the exponents, we calculate the derived functions: 1$)$ the 'fluxes' $\varphi(x ; \alpha)=a(x ; \alpha) \phi^{\prime}(x ; \alpha)$

$$
\left\{\begin{aligned}
\varphi_{j}(x ; \alpha) & =+\operatorname{sign}(x) \omega_{j}|x|^{\omega_{j}-1+\alpha}, \\
\varphi_{j+2}(x ; \alpha) & =-\operatorname{sign}(x) \omega_{j+2}|x|^{\omega_{j+2}-1+\alpha},
\end{aligned}\right.
$$

and 2) their derivatives $\psi(x ; \alpha)=\varphi^{\prime}(x ; \alpha)=\left[a(x ; \alpha) \phi^{\prime}(x ; \alpha)\right]^{\prime}$

$$
\left\{\begin{aligned}
\psi_{j}(x ; \alpha) & =+\omega_{j}\left[\omega_{j}-\theta+1\right]|x|^{\omega_{j}-\theta} \\
\psi_{j+2}(x ; \alpha) & =-\omega_{j+2}\left[\omega_{j+2}-\theta+1\right]|x|^{\omega_{j+2}-\theta}
\end{aligned}\right.
$$

and assume that they vanish at $x=0$ smoothly, i. e., $\psi_{j}(0 ; \alpha)=\psi_{j+2}(0 ; \alpha)=0$ and $\psi_{j}^{\prime}(0 ; \alpha)=\psi_{j+2}^{\prime}(0 ; \alpha)=0$, whence we immediately deduce that $\omega_{j}-\theta=1+\epsilon_{j}$, $\omega_{j+2}-\theta=1+\epsilon_{j+2}$, where $\epsilon_{j}, \epsilon_{j+2}>0$.

Taking $\omega_{j}=\omega_{j+2}=\omega$ and substituting the functions $\phi_{j}(x ; \alpha), \phi_{j+2}(x ; \alpha)(4.7)$ in the coefficients $(2.49),(2.50)$ yields to

$$
\left\{\begin{array}{l}
a_{\mu}(\alpha)=-\frac{1}{\left\|Z_{1, \mu}\right\|^{2}} I_{2}(\alpha, \omega) \\
b_{\mu}(\alpha)=+\frac{\vartheta}{\left\|Z_{1, \mu}\right\|^{2}} I_{1}(\alpha, \omega) \\
c_{\mu}(\alpha)=-\frac{1}{\left\|Z_{1, \mu}\right\|^{2}} I_{0}(\alpha)+\frac{1}{\left\|Z_{1, \mu}\right\|^{2}} I_{2}(\alpha, \omega) \\
d_{\mu}(\alpha)=-\frac{\vartheta}{\left\|Z_{1, \mu}\right\|^{2}} I_{1}(\alpha, \omega) .
\end{array}\right.
$$

The definite integrals $I_{0}(\alpha), I_{1}(\alpha, \omega), I_{2}(\alpha, \omega)$ in (4.10) can be calculated ana- 
lytically, applying the variable transformation $s=s x^{\frac{\theta}{2}}$ as follows

$$
\left\{\begin{aligned}
I_{0}(\alpha) & =\int_{0}^{1} Z_{1, \mu}(x ; \alpha) d x= \\
& =\frac{2}{\theta}\left(\frac{1}{s}\right)^{o+1} \int_{0}^{s} s^{o} \mathrm{Z}_{\varrho}(s) d s \equiv \frac{2}{\theta}\left(\frac{1}{s}\right)^{o+1} I_{0}^{*}(\alpha), \\
I_{1}(\alpha, \omega) & =\int_{0}^{1} x^{\omega-\theta} Z_{1, \mu}(x ; \alpha) d x=\int_{0}^{1} x^{\epsilon+1} x^{\frac{\nu}{2}} \mathrm{Z}_{\varrho}(s) d x \\
& =\frac{2}{\theta}\left(\frac{1}{s}\right)^{v+1} \int_{0}^{s} s^{v} \mathrm{Z}_{\varrho}(s) d s \equiv \frac{2}{\theta}\left(\frac{1}{s}\right)^{v+1} I_{1}^{*}(\alpha, \epsilon), \\
I_{2}(\alpha, \omega) & =\int_{0}^{1} x^{\omega} Z_{1, \mu}(x ; \alpha) \mathrm{d} x=\int_{0}^{1} x^{\theta+\epsilon+1} x^{\frac{\nu}{2}} \mathrm{Z}_{\varrho}(s) d x \\
& =\frac{2}{\theta}\left(\frac{1}{s}\right)^{v+3} \int_{0}^{s} s^{v+2} \mathrm{Z}_{\varrho}(s) d s \equiv \frac{2}{\theta}\left(\frac{1}{s}\right)^{v+3} I_{2}^{*}(\alpha, \epsilon),
\end{aligned}\right.
$$

where

$$
o=\frac{1}{\theta}, \quad v=\frac{2 \epsilon+3}{\theta},
$$

and to avoid confusion with zero, small letter ' $\mathrm{o}$ ' is used only for the exponents, and the notation $\stackrel{s}{s}=s_{1, \mu}$ is used hereinafter to present calculations of the transformed integrals $I_{0}^{*}(\alpha), I_{1}^{*}(\alpha)$ and $I_{2}^{*}(\alpha, \epsilon)$ in a compact form.

We chose the analytical approach to calculate the integrals in (4.11), hence, our concern is calculating the transformed integrals

$$
\left\{\begin{array}{l}
I_{0}^{*}(\alpha)=\int_{0}^{\stackrel{s}{s}} s^{o} \mathrm{Z}_{\varrho}(s) d s \\
I_{1}^{*}(\alpha, \epsilon)=\int_{0}^{\stackrel{s}{s}} s^{v} \mathrm{Z}_{\varrho}(s) d s \\
I_{2}^{*}(\alpha, \epsilon)=\int_{0}^{\stackrel{s}{s}} s^{v+2} \mathrm{Z}_{\varrho}(s) d s
\end{array}\right.
$$

using the following recurrence formula [7]

$$
s^{\varrho+1} \mathrm{Z}_{\varrho}(s)=\left[s^{\varrho+1} \mathrm{Z}_{\varrho+1}(s)\right]^{\prime} .
$$

The integral $I_{0}^{*}(\alpha)$ is easily shown to be calculated exactly for any $\alpha$. Indeed, keeping in mind (4.14), we obtain that

$$
\left\{\begin{aligned}
I_{0}^{*}(\alpha) & =\int_{0}^{s} s^{o} \mathrm{Z}_{\varrho}(s) d s=\int_{0}^{s} s^{\varrho+1} \mathrm{Z}_{\varrho}(s) d s \\
& =\int_{0}^{s}\left[s^{\varrho+1} \mathrm{Z}_{\varrho+1}(s)\right]^{\prime} d s=s^{\varrho+1} \mathrm{Z}_{\varrho+1}(\stackrel{\circ}{s}) .
\end{aligned}\right.
$$


To calculate the transformed integrals $I_{1}^{*}(\alpha ; \epsilon), I_{2}^{*}(\alpha ; \epsilon)$ (4.13), we introduce the following

Definition 4.1. The values of the parameter $\epsilon>0$ in the exponents $\omega=\theta+1+\epsilon$ (4.7), (4.9), allowing for: 1) the function (4.9) to be continuously differentiable and 2) the transformed integrals $I_{1}^{*}(\alpha, \epsilon), I_{2}^{*}(\alpha, \epsilon)(4.13)$ to be calculated by parts (this is referred to as integrability), are called proper.

Proposition 4.1. The proper values of the parameter $\epsilon$ are the positive values produced by the formula

$$
\epsilon=-1+k \theta=-1+k(2-\alpha),
$$

where $k \in \mathbb{N}$.

Proof. First, we find the values of the exponent $v$ leading to integration by parts using the following formula (4.14). Presenting the exponent of the integrand of $I_{2}^{*}$ as $v=v-\varrho-1+(\varrho+1)=v^{\prime}+(\varrho+1)$ makes it clear that: 1$)$ it is the term $v^{\prime} \geqslant 0$ that is responsible for integrability; 2$) v^{\prime} \equiv 0(\bmod 2)$ is the integrability condition. Indeed, let:

a) $v^{\prime}=0$, then the integral $I_{2}^{*}$ reads

$$
I_{2}^{*}(\alpha, \epsilon)=\int_{0}^{\stackrel{\varsigma}{s}} s^{v^{\prime}}\left[s^{\varrho+1} \mathrm{Z}_{\varrho}(s)\right] d s=\int_{0}^{\stackrel{\varsigma}{s}}\left[s^{\varrho+1} \mathrm{Z}_{\varrho+1}(s)\right]^{\prime} d s=s^{\varrho+1} \mathrm{Z}_{\varrho+1}(\stackrel{\circ}{)}) ;
$$

b) $v^{\prime}=2$, then integration by parts is performed successfully as well

$$
\begin{aligned}
& I_{2}^{*}(\alpha, \epsilon)=\int_{0}^{\stackrel{\varrho}{s}} s^{v^{\prime}}\left[s^{\varrho+1} \mathrm{Z}_{\varrho}(s)\right] d s=\int_{0}^{\stackrel{\varrho}{s}} s^{2}\left[s^{\varrho+1} \mathrm{Z}_{\varrho+1}(s)\right]^{\prime} d s \\
& =s^{\varrho+3} \mathrm{Z}_{\varrho+1}(\stackrel{\circ}{s})-2 \int_{0}^{\stackrel{\wp}{s}} s^{\varrho+2} \mathrm{Z}_{\varrho+1}(s) d s
\end{aligned}
$$

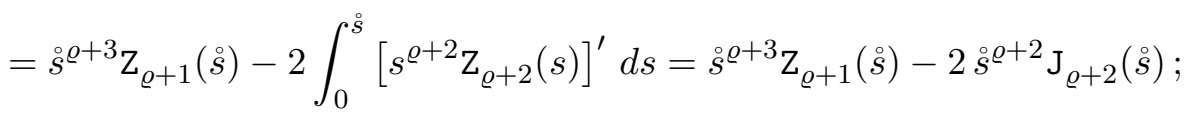

c) $v^{\prime}=4$, then integration is reduced to the previous case

$$
\begin{aligned}
& I_{2}^{*}(\alpha, \epsilon)=\int_{0}^{\stackrel{\wp}{s}} s^{4}\left[s^{\varrho+1} \mathrm{Z}_{\varrho+1}(s)\right]^{\prime} d s=s^{\varrho+5} \mathrm{Z}_{\varrho+1}(\stackrel{\bullet}{s})-4 \int_{0}^{\stackrel{\wp}{s}} s^{2}\left[s^{\varrho+3} \mathrm{Z}_{\varrho+1}(s)\right] d s \\
& =s^{\varrho+5} \mathrm{Z}_{\varrho+1}\left(\stackrel{\Im}{)}-4 \int_{0}^{\stackrel{s}{s}} s^{2}\left[s^{\varrho+3} \mathrm{Z}_{\varrho+3}(s)\right]^{\prime} d s,\right.
\end{aligned}
$$

..., etc. It is evident that no value of the exponent leading to integrability other than those indicated above exists.

Second, considering the integral $I_{1}^{*}$ is performed exactly in the same way as the integral $I_{2}^{*}$. 
Third, we gather our observations on integrability as the following condition imposed on $v^{\prime}: v^{\prime}=2 k, k \in \mathbb{Z}_{+}$, or reformulated for $v$ as follows

$$
v-\varrho-1=2 k, \quad k \in \mathbb{Z}_{+},
$$

and substituting the expressions for $v(4.12)$ and $\varrho(2.3)$ in the above condition we obtain

$$
\frac{2 \epsilon+3}{2-\alpha}+\frac{1-\alpha}{2-\alpha}-1=2 \frac{\epsilon+1}{2-\alpha}=2 k .
$$

Resolving the above condition with respect to $\epsilon$ yields to (4.16). It is evident, that zero value of $k$ produces the value $\epsilon=-1$ and must be neglected. Unfortunately, other values of $k$ produce negative values of the parameter $\epsilon$ as well, indeed: a) for $k=1$ we obtain $\epsilon=1-\alpha ; b)$ for $k=2$ it yields to $\epsilon=3-2 \alpha$, etc., therefore (4.16) needs to be adjusted as the proposition says.

The 1-parameter family (4.2) of the admissible values of $\epsilon$ is shown in Fig. 4.2.

We show below, how the transformed integrals $I_{1}^{*}$ and $I_{2}^{*}$ (4.13) can be calculated exactly for non-unique proper values of the parameter $\epsilon$, choosing the following values of the parameter $\alpha$ : 1) $\left.\frac{1}{2}, 2\right) 1$, and 3) $\frac{3}{2}$.

1) Let $\alpha=\frac{1}{2}$, then $\theta=\frac{3}{2}, \nu=\frac{1}{2}, \varrho=-\frac{1}{3}$, and $v=\frac{4}{3} \epsilon+2$ (4.12), and applying the property (4.14) yields to

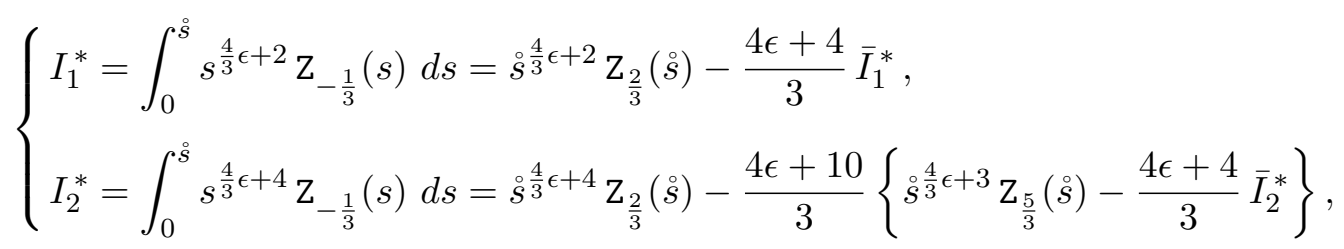

where the integrals $\bar{I}_{1}^{*}$ and $\bar{I}_{2}^{*}$ are

$$
\left\{\begin{array}{l}
\bar{I}_{1}^{*}=\int_{0}^{\stackrel{\diamond}{s}} s^{\frac{4}{3} \epsilon-\frac{2}{3}} s^{\frac{5}{3}} \mathrm{Z}_{\frac{2}{3}}(s) d s \\
\bar{I}_{2}^{*}=\int_{0}^{\stackrel{\circ}{s}} s^{\frac{4}{3} \epsilon-\frac{2}{3}} s^{\frac{8}{3}} \mathrm{Z}_{\frac{5}{3}}(s) d s .
\end{array}\right.
$$

Assuming that $\epsilon=\frac{1}{2}$, we easily find that

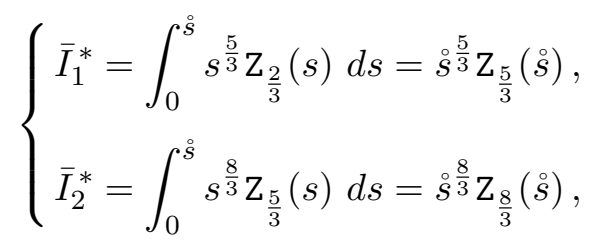

and complete the calculating of the integrals $I_{1}^{*}$ and $I_{2}^{*}$ as follows

$$
\left\{\begin{array}{l}
I_{1}^{*}=s^{\frac{8}{3}} \mathrm{Z}_{\frac{2}{3}}\left(s^{\circ}\right)-2 s^{\frac{5}{3}} \mathrm{Z}_{\frac{5}{3}}\left(s^{\circ}\right), \\
I_{2}^{*}=s^{\frac{14}{3}} \mathrm{Z}_{\frac{2}{3}}(s)-4 s^{\frac{11}{3}} \mathrm{Z}_{\frac{5}{3}}(s)+8 s^{\frac{8}{3}} \mathrm{Z}_{\frac{8}{3}}(s),
\end{array}\right.
$$




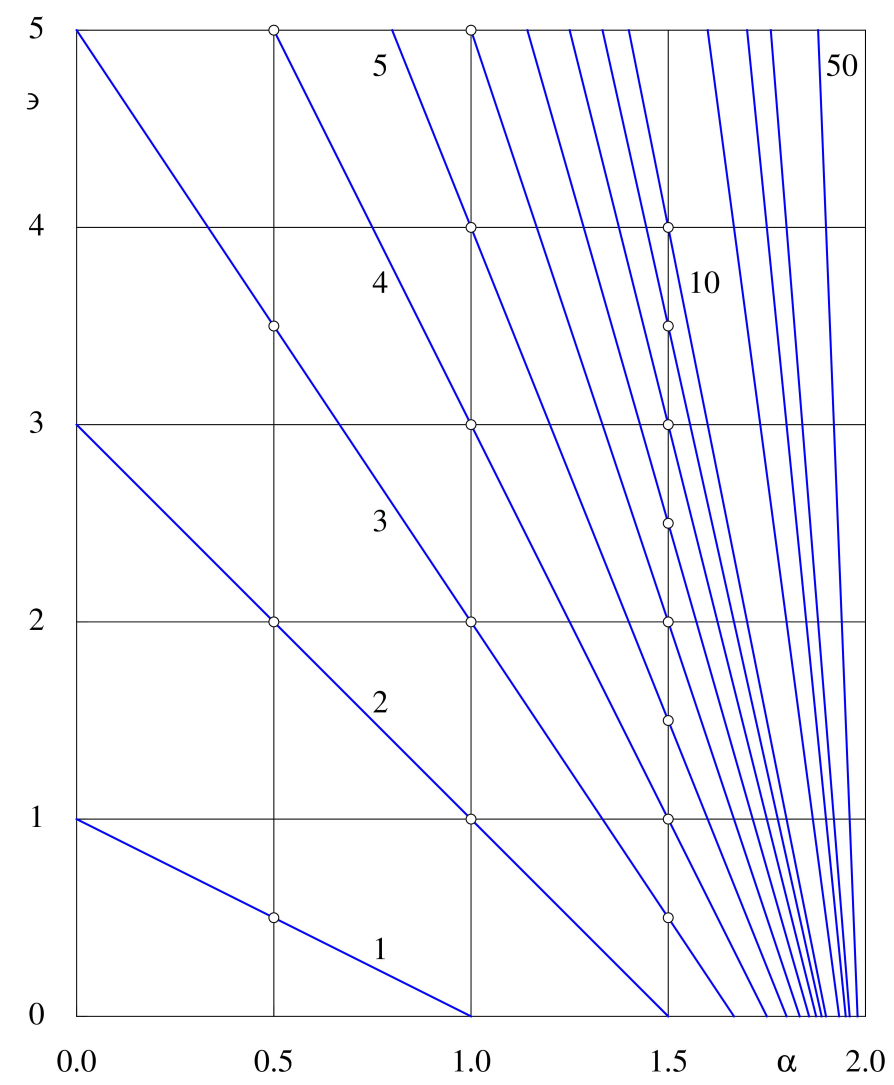

Fig. 4.2. The proper values of $\epsilon$ vs $\alpha$ (4.16) for various values of the parameter $k$ : $k=1(1) 10 ; 15,20,25,50$. Multiple proper values of $\epsilon$ for $\alpha=0.5,1.0,1.5$ are marked with the white disks. The upper side of the 1-parameter family (4.16) is cut off along the straight line $\epsilon=5$

then, assuming that $\epsilon=2$

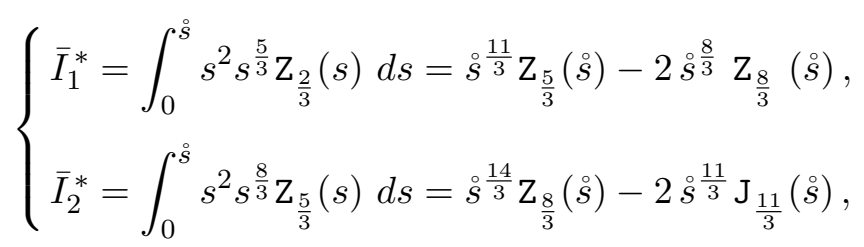

and complete the calculation of the integrals $I_{1}^{*}$ and $I_{2}^{*}$ as follows

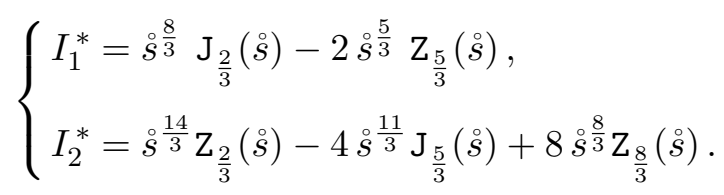

2) Let $\alpha=1$, then $\theta=1, \nu=0, \varrho=0$, and $v=2 \epsilon+3$ (4.12), and applying 
the property (4.14) yields to

$$
\left\{\begin{array}{l}
I_{1}^{*}=\int_{0}^{s} s^{2 \epsilon+2}\left[s \mathrm{Z}_{0}(s)\right] d s=s^{2 \epsilon+3} \mathrm{Z}_{0}(\stackrel{\circ}{s})-(2 \epsilon+2) \bar{I}_{1}^{*}, \\
I_{2}^{*}=\int_{0}^{s} s^{2 \epsilon+4}\left[s \mathrm{Z}_{0}(s)\right] d s=\stackrel{s}{s}^{2 \epsilon+5} \mathrm{Z}_{0}(\stackrel{\circ}{s})-(2 \epsilon+4)\left\{\stackrel{s}{s}^{2 \epsilon+4} \mathrm{Z}_{2}\left(s^{\circ}\right)-(2 \epsilon+2) \bar{I}_{2}^{*}\right\},
\end{array}\right.
$$

where the integrals $\bar{I}_{1}^{*}$ and $\bar{I}_{2}^{*}$ are

$$
\left\{\begin{array}{l}
\bar{I}_{1}^{*}=\int_{0}^{\stackrel{\diamond}{s}} s^{2 \epsilon}\left[s^{2} \mathrm{Z}_{2}(s)\right]^{\prime} d s \\
\bar{I}_{2}^{*}=\int_{0}^{\stackrel{\diamond}{s}} s^{2 \epsilon}\left[s^{3} \mathrm{Z}_{3}(s)\right]^{\prime} d s
\end{array}\right.
$$

Assuming that $\epsilon=1$, we easily calculate both integrals $\bar{I}_{1,2}^{*}$

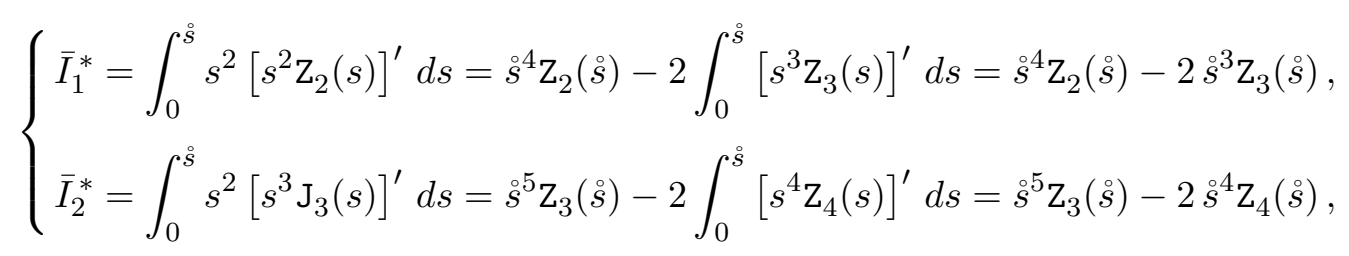

and eventually find

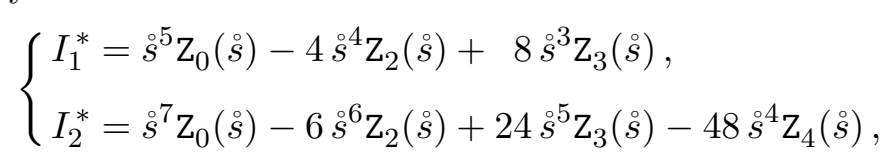

whereas assuming that $\epsilon=2$, we find both integrals $\bar{I}_{1,2}^{*}$ to equal

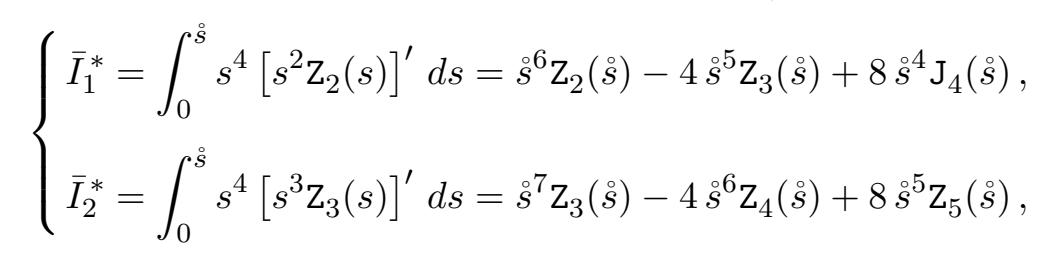

yielding to

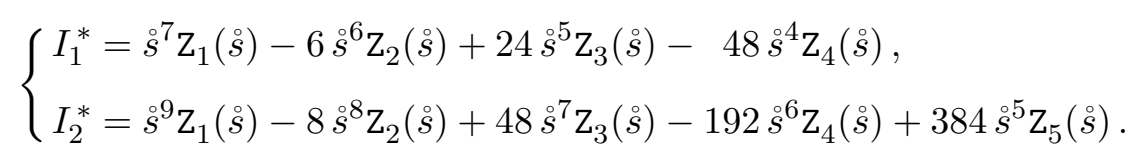

3) Let $\alpha=\frac{3}{2}$, then $\theta=\frac{1}{2}, \nu=-\frac{1}{2}, \varrho=1$, and $v=4 \epsilon+6$ (4.12), and applying the property (4.14) yields to

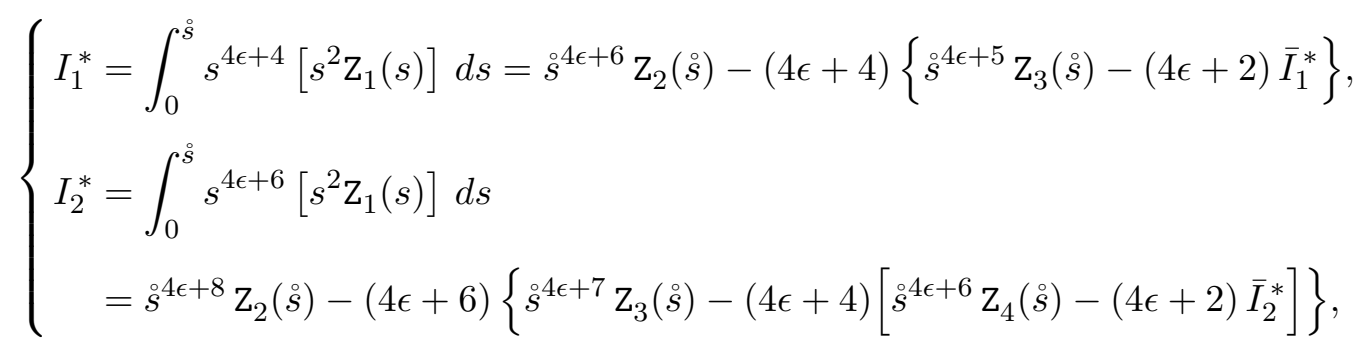


where the integrals $\bar{I}_{1}^{*}$ and $\bar{I}_{2}^{*}$ are

$$
\left\{\begin{array}{l}
\bar{I}_{1}^{*}=\int_{0}^{s} s^{4 \epsilon}\left[s^{4} \mathrm{Z}_{4}(s)\right]^{\prime} d s \\
\bar{I}_{2}^{*}=\int_{0}^{s} s^{4 \epsilon}\left[s^{5} \mathrm{Z}_{5}(s)\right]^{\prime} d s
\end{array}\right.
$$

Assumption $\epsilon=\frac{1}{2}$ gives

$$
\left\{\begin{array}{l}
\bar{I}_{1}^{*}=\int_{0}^{s} s^{2}\left[s^{4} \mathrm{Z}_{4}(s)\right]^{\prime} d s=s^{6} \mathrm{Z}_{4}(\stackrel{\circ}{s})-2 s^{\circ} \mathrm{Z}_{5}\left(s^{\circ}\right) \\
\bar{I}_{2}^{*}=\int_{0}^{s} s^{2}\left[s^{5} \mathrm{Z}_{5}(s)\right]^{\prime} \mathrm{d} s=s^{7} \mathrm{Z}_{5}(\stackrel{\circ}{s})-2 s^{\circ} \mathrm{Z}_{6}(\stackrel{\circ}{s})
\end{array}\right.
$$

that yields to

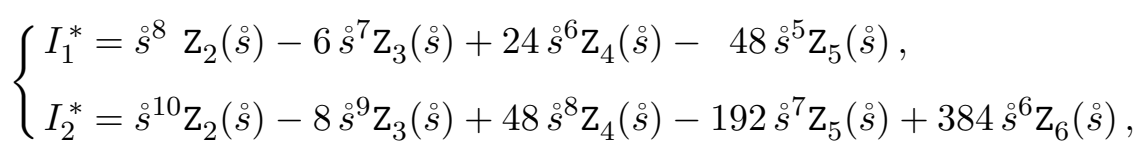

whereas assumption $\epsilon=1$ leads to

$$
\left\{\begin{array}{l}
\bar{I}_{1}^{*}=\int_{0}^{s} s^{4}\left[s^{4} \mathrm{~J}_{4}(s)\right]^{\prime} d s=s^{8} \mathrm{~J}_{4}(\stackrel{\circ}{s})-4 s^{7} \mathrm{~J}_{5}(\stackrel{\circ}{s})+8 s^{\circ} \mathrm{J}_{6}\left({ }^{\circ}\right), \\
\bar{I}_{2}^{*}=\int_{0}^{s} s^{4}\left[s^{5} \mathrm{~J}_{5}(s)\right]^{\prime} d s=s^{9} \mathrm{~J}_{5}(\stackrel{\circ}{s})-4 s^{8} \mathrm{~J}_{6}(\stackrel{\circ}{s})+8 s^{7} \mathrm{~J}_{7}(\stackrel{\circ}{s}),
\end{array}\right.
$$

and eventually to

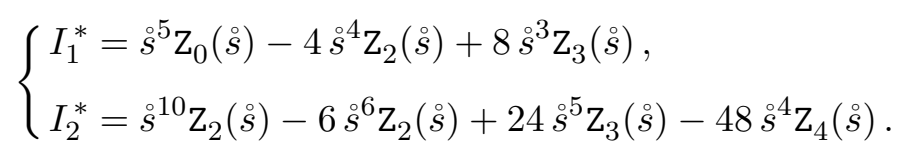

As an example, we choose the values $\alpha=1(\varrho=0, \theta=1)$ and $\epsilon=1$, then substitute the formula (4.15) and the values (4.17) of $I_{1}^{*}, I_{2}^{*}$ into the expressions (4.11) to obtain

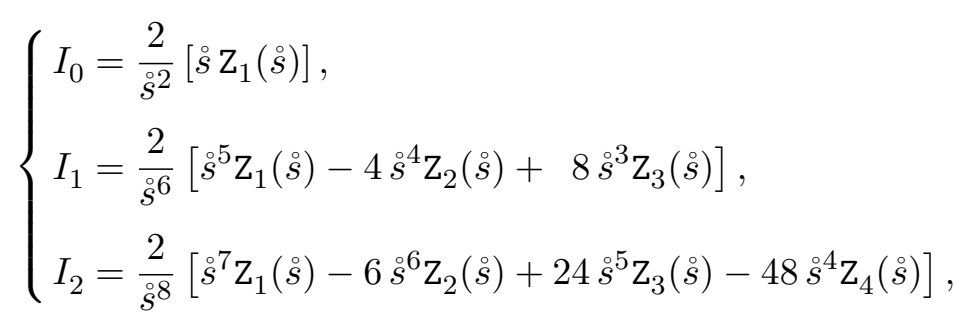


and eventually find the required coefficients (4.10)

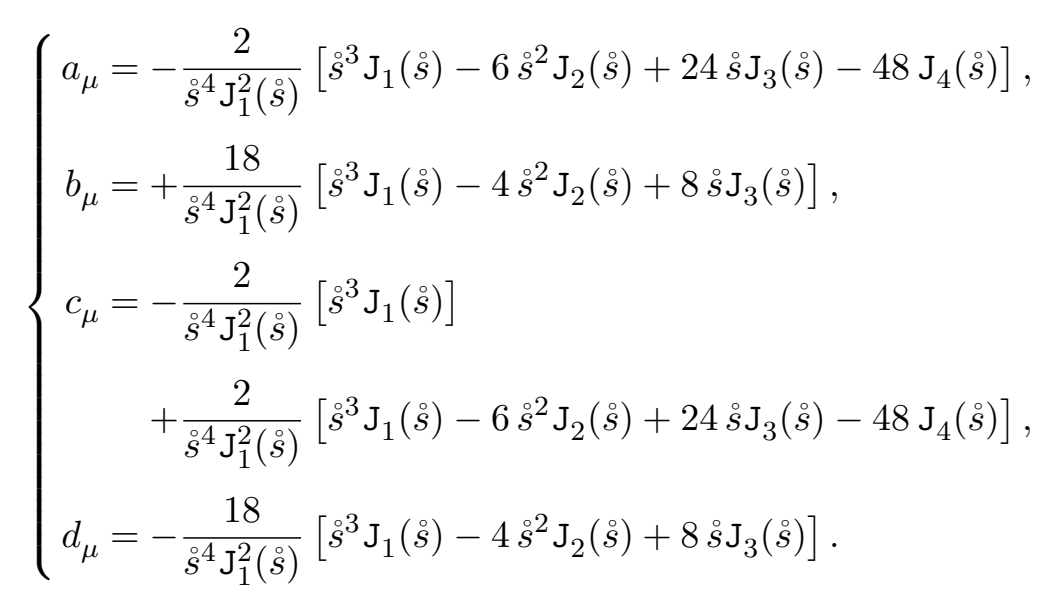

\section{References}

1. V.L. Bonsch, On initial boundary value problems for the degenerate $1 D$ wave equation, Journal of Optimization, Differential Equations, and their Applications (JODEA), 27(2) (2019), 27-44.

2. V. L. Borsch, P. I. Kogut, G. Leugering, On an initial boundary-value problem for $1 D$ hyperbolic equation with interior degeneracy: series solutions with the continuously differentiable fluxes, Journal of Optimization, Differential Equations, and their Applications (JODEA), 28(1) (2020), 1-42.

3. G. DoETsCh, Introduction to the Theory and Application of the Laplace Transformation, Springer, NY, 1974.

4. A. M. Kohen, Numerical Methods for Laplace Transform Inversion, Springer Science+Business Media, LLC, NY, 2007.

5. Yu. V. Sidorov, M. V. Fedoryuk, M. I. Shabunin, Lectures on the Theory of Functions of a Complex Variable, Mir Publishers, Moscow, 1985.

6. G. P. Tolstov, Fourier Series, Dover, NY, 1962.

7. G. N. Watson, A Treatise on the Theory of Bessel Functions, Cambridge University Press, Cambridge, 1922. 